Groups Geom. Dyn. 6 (2012), 441-483

DOI $10.4171 / \mathrm{GGD} / 163$
Groups, Geometry, and Dynamics

(C) European Mathematical Society

\title{
Anosov AdS representations are quasi-Fuchsian
}

\author{
Thierry Barbot* and Quentin Mérigot
}

\begin{abstract}
Let $\Gamma$ be a cocompact lattice in $\mathrm{SO}(1, n)$. A representation $\rho: \Gamma \rightarrow \mathrm{SO}(2, n)$ is called quasi-Fuchsian if it is faithful, discrete, and preserves an acausal subset in the boundary of anti-de Sitter space. A special case are Fuchsian representations, i.e., compositions of the inclusions $\Gamma \subset \mathrm{SO}(1, n)$ and $\mathrm{SO}(1, n) \subset \mathrm{SO}(2, n)$. We prove that quasi-Fuchsian representations are precisely those representations which are Anosov in the sense of Labourie (cf. [Lab06]). The study involves the geometry of locally anti-de Sitter spaces: quasi-Fuchsian representations are holonomy representations of globally hyperbolic spacetimes diffeomorphic to $\mathbb{R} \times \Gamma \backslash \mathbb{H}^{n}$ locally modeled on $\mathrm{AdS}_{n+1}$.
\end{abstract}

Mathematics Subject Classification (2010). 53C50, 53C15, $20 \mathrm{H} 12$.

Keywords. Globally hyperbolic AdS spacetimes, Anosov representations.

\section{Contents}

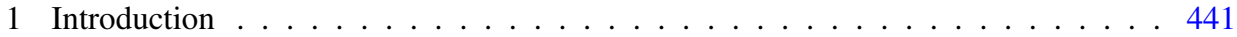

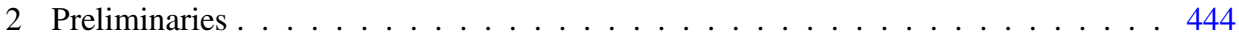

3 Regular AdS manifolds . . . . . . . . . . . . . . . . . . . . . . . . . . . . . . . . 449

4 Globally hyperbolic AdS spacetimes . . . . . . . . . . . . . . . . . 455

5 Anosov representations are strictly GHC-regular . . . . . . . . . . . . . . . . . . . . . . . . . . . . . . . . . . .

6 Dynamical properties . . . . . . . . . . . . . . . . . 465

7 Convex hull of GHC-representations . . . . . . . . . . . . . . . . 468

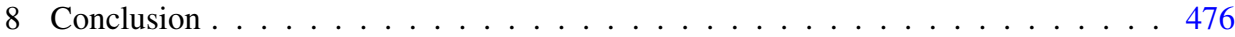

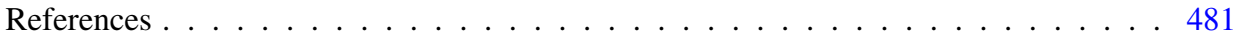

\section{Introduction}

Let $\mathrm{SO}_{0}(1, n), \mathrm{SO}_{0}(2, n)$ denote the identity components of respectively $\mathrm{SO}(1, n)$, $\mathrm{SO}(2, n)$. Let $\Gamma$ be a cocompact torsion free lattice in $\mathrm{SO}_{0}(1, n)$. For any Lie group $G$ let $\operatorname{Rep}(\Gamma, G)$ denote the space of representations of $\Gamma$ into $G$ equipped with the compact-open topology.

\footnotetext{
* Supported by ANR project GEODYCOS.
} 
In the case $G=\mathrm{SO}_{0}(1, n+1)$ we distinguish the Fuchsian representations: they are the representations obtained by composition of an embedding $\Gamma \subset \operatorname{SO}_{0}(1, n)$ and any faithful representation of $\mathrm{SO}_{0}(1, n)$ into $\mathrm{SO}_{0}(1, n+1)$. Their characteristic property is to be faithful, discrete, and to preserve a totally geodesic copy of $\mathbb{H}^{n}$ into $\mathbb{H}^{n+1}$. The boundary of the $\rho_{0}(\Gamma)$-invariant totally geodesic hypersurface $\mathbb{H}^{n} \subset$ $\mathbb{H}^{n+1}$ provides such a topological sphere.

We can relax this last condition by only requiring the existence of a $\rho(\Gamma)$-invariant topological $(n-1)$-sphere in $\partial \mathbb{H}^{n+1}$, thus defining the notion of quasi-Fuchsian representation. We denote by $\mathcal{Q} \mathcal{F}\left(\Gamma, \mathrm{SO}_{0}(1, n+1)\right)$ the set of quasi-Fuchsian representations. It is well known that $\mathcal{Q} \mathscr{F}\left(\Gamma, \mathrm{SO}_{0}(1, n+1)\right)$ is a neighborhood of Fuchsian representations in the space of representations of $\Gamma$ into $\operatorname{SO}_{0}(1, n+1)$, and this assertion can be proven using the Anosov character of the geodesic flow $\Phi^{t}$ of the hyperbolic manifold $N=\Gamma \backslash \mathrm{T}^{1} \mathbb{H}^{n}$ (for definitions, see $\S 5.1 .1$ ).

This kind of argument has been extended in a more general framework by F. Labourie in [Lab06]. He defined, for any pair $(G, Y)$ where $G$ is a Lie group acting on a manifold $Y$, the notion of $(G, Y)$-Anosov representation (or simply Anosov representation when there is no ambiguity about the pair $(G, Y))$. We denote this space of representation by $\operatorname{Anos}_{Y}(\Gamma, G)$ (see $\S 5.1 .1$ ). By structural stability, $\operatorname{Anos}_{Y}(\Gamma, G)$ is an open domain; simple and general arguments ensure that Anosov representations are faithful, with a discrete image formed by the loxodromic elements. As a matter of fact, the sets of quasi-Fuchsian representations $\mathcal{Q} \mathcal{F}\left(\Gamma, \mathrm{SO}_{0}(1, n+1)\right)$ and Anosov representations $\operatorname{Anos}_{Y}\left(\Gamma, \mathrm{SO}_{0}(1, n+1)\right)$ coincide, where $Y=\partial \mathbb{H}^{n+1} \times \partial \mathbb{H}^{n+1} \backslash \mathfrak{D}$. Observe however that the fact that quasi-Fuchsian representations are Anosov is not already obvious - it can, in fact, be obtained by adapting the arguments given in the present paper.

Anosov representations have been studied in different situations, mostly in the case $n=2$, i.e., the case where $\Gamma$ is a surface group:

In [Lab06], F. Labourie considered the case where $G$ is the group $\operatorname{SL}(n, \mathbb{R})$ and $Y$ the frame variety. He proved that one of the connected components of $\operatorname{Anos}_{Y}(\Gamma, G)$, the quasi-Fuchsian component, coincides with the Hitchin component of component of $\operatorname{Rep}(\Gamma, G)$. Moreover, he proved that these quasi-Fuchsian representations are hyperconvex, i.e., that they preserve some curve in the projective space $\mathbb{P}\left(\mathbb{R}^{n}\right)$ with some very strong convexity properties. In [Gui08], O. Guichard then proved that conversely hyperconvex representations are quasi-Fuchsian. Note however that $(G, Y)$-Anosov representations are not necessarily quasi-Fuchsian; in other words, $\operatorname{Anos}_{Y}(\Gamma, G)$ is not connected. See [Bar10].

In [BLIW05], the authors also used the notion of Anosov representations for the study of representations of surface groups into the symplectic group of a real symplectic vector space with maximal Toledo invariant.

In the present paper we consider the case where $\Gamma$ is a cocompact lattice of $\mathrm{SO}_{0}(1, n)$ that we deform in $G=\mathrm{SO}_{0}(2, n)$. While in the case of quasi-Fuchsian representations into $\mathrm{SO}_{0}(1, n+1)$ presented above the geometry of the hyperbolic space played an important role, the study of $\operatorname{Rep}\left(\Gamma, \mathrm{SO}_{0}(2, n)\right)$ deeply involves the 
geometry of the Lorentzian analog of this space, i.e., the anti-de Sitter space $\operatorname{AdS}_{n+1}$. In Lorentzian geometry appear some phenomena, latent in the Riemannian context, related to the causality notions. While in the hyperbolic space a pair of points is only distinguished by their mutual distance, in the anti-de Sitter space we have to distinguish three types of pairs of points, according to the nature of the geodesic joining the two points: this geodesic may be space-like, light-like or time-like - in the last two cases the points are said causally related.

The anti-de Sitter space $\mathrm{AdS}_{n+1}$ admits a conformal boundary called the Einstein universe and denoted by $\operatorname{Ein}_{n}$, which plays a role similar to that of the conformal boundary $\partial \mathbb{H}^{n+1}$ for the hyperbolic space. The Einstein universe is a conformal Lorentzian spacetime and is also subject to a causality notion: in particular, a subset $\Lambda$ of the Einstein space $\operatorname{Ein}_{n}$ is called acausal if the unique geodesic in $\operatorname{AdS}_{n+1}$ between any pair of distinct points in $\Lambda$ is space-like. Finally, a representation $\rho: \Gamma \rightarrow \mathrm{SO}_{0}(2, n)$ is quasi-Fuchsian if it preserves an acausal topological $(n-1)$ sphere in $\operatorname{Ein}_{n}$.

In the following theorem, $y$ denotes the subset of $\operatorname{Ein}_{n} \times \operatorname{Ein}_{n}$ made of noncausally related pairs, i.e., pairs of points that can be joined by a space-like geodesic in $\operatorname{AdS}_{n+1}$.

Theorem 1.1. A representation $\rho: \Gamma \rightarrow \mathrm{SO}_{0}(2, n)$ is quasi-Fuchsian if and only if it is $\left(\mathrm{SO}_{0}(2, n), \mathcal{Y}\right)$-Anosov.

The geometric ingredient of this Theorem is the characterization of quasi-Fuchsian representations as holonomy representations of Lorentzian manifolds locally modelled on anti-de Sitter which are spatially compact globally hyperbolic (GHC).

Let us remind a few classical definitions in Lorentzian geometry. A tangent vector $v$ in a Lorentzian manifold $(M, g)$ is called time-like (resp. light-like, causal and space-like) if $g(v, v)$ is negative (resp. null, non-positive and positive). A smooth curve whose tangent vectors all have the same sign is called with the same name; it is called inextendible if it is maximal for the inclusion over smooth curves with the same type. By spacetime we mean here an oriented Lorentzian manifold with a time orientation given by a smooth time-like vector field. This allows to define the notion of future and past-directed causal curves.

A spacetime $(M, g)$ is globally hyperbolic (abbreviated to $\mathrm{GH}$ ) if it admits a Cauchy hypersurface, that is an achronal set $S$ which intersects every inextendible time-like curve at exactly one point. This set is then automatically a locally Lipschitz hypersurface (see [O’N83], § 14, Lemma 29). A globally hyperbolic spacetime is strongly causal in the sense that its topology admits a basis of causally convex neighborhoods, i.e., neighborhoods $U$ such that any causal curve with extremities in $U$ is contained in $U$. However, the converse implication is false in general. A globally hyperbolic spacetime is called spatially compact if its Cauchy hypersurfaces are compact. It is equivalent to require the existence of a proper time function, i.e., a proper real-valued function that is strictly increasing along future-directed causal curves. 
It turns out that Theorem 1.1 is merely a particular case of the following theorem, where $\Gamma$ is not necessarily a lattice in $\operatorname{SO}_{0}(1, n)$. Here a representation $\rho$ is called strictly GHC-regular if $\rho(\Gamma)$ preserves an acausal topological $(n-1)$-sphere $\Lambda$ in the Einstein boundary $\operatorname{Ein}_{n}$ of the anti-de Sitter space $\operatorname{AdS}_{n+1}$.

Theorem 1.2. Let $\Gamma$ be the fundamental group of a negatively curved closed Riemannian manifold. Then, a representation $\rho: \Gamma \rightarrow \mathrm{SO}_{0}(2, n)$ is strictly GHC-regular if and only if it is $\left(\mathrm{SO}_{0}(2, n), y\right)$-Anosov.

Overview of the paper. In $\S 2$ we review some geometric properties of anti-de Sitter and Einstein spaces. In $\S 3$ we introduce the notion of regular AdS domains. In $\S 4$ we exhibit the link between AdS regular manifolds and globally hyperbolic AdS manifolds. In $\S 5$ we define the notion of Anosov representations. In this section, and more precisely in $\S 5.3$, we prove one half of Theorem 1.2, namely that $\left(\mathrm{SO}_{0}(2, n), y\right)$-representations are strictly GHC-regular. The proof of the theorem is completed in $\S 7.5$, where we show the reverse implication (strictly GHC-regular representations are $\left(\mathrm{SO}_{0}(2, n), y\right)$-Anosov $)$. For that purpose we develop in $\S 6$ a theory of dynamics of sequences in $\mathrm{SO}_{0}(2, n)$, and in $\S 7$ a detailed study of the geometry of convex hulls of acausal topological spheres in $\operatorname{Ein}_{n}$ is carried out. It includes a generalization of Dirichlet domains of discrete groups of isometry in the AdS background.

In $\S 8$ we conclude by addressing some questions in relation to what we expect to be the classification of globally hyperbolic AdS manifolds. In particular, we expect that the "negatively curved" hypothesis appearing in Theorem 1.2 can be removed, and moreover, that strictly GHC-regular representations are presumably all quasiFuchsian.

\section{Preliminaries}

We assume the reader acquainted to basic causality notions in Lorentzian manifolds like causal or time-like curves, inextendible causal curves, time orientation, future and past of subsets, time function, achronal subsets, etc. We refer to [BEE96] or [O’N83], § 14, for further details.

2.1. Anti-de Sitter space. Let $\mathbb{R}^{2, n}$ be the vector space of dimension $n+2$, with coordinates $\left(u, v, x_{1}, \ldots, x_{n}\right)$, and endowed with the quadratic form:

$$
\mathrm{q}_{2, n}\left(u, v, x_{1}, \ldots, x_{n}\right):=-u^{2}-v^{2}+x_{1}^{2}+\cdots+x_{n}^{2} .
$$

We denote by $\langle x \mid y\rangle$ the associated scalar product. For any subset $A$ of $\mathbb{R}^{2, n}$ let $A^{\perp}$ be the orthogonal of $A$, i.e., the set of elements $y$ in $\mathbb{R}^{2, n}$ such that $\langle y \mid x\rangle=0$ for every $x$ in $A$. The isotropic cone $\left\{w \in \mathbb{R}^{2, n} \mid \mathrm{q}_{2, n}(w)=0\right\}$ is denoted by $\bigodot_{n}$. 
Definition 2.1. The hypersurface $\left\{w \in \mathbb{R}^{2, n} \mid \mathrm{q}_{2, n}(w)=-1\right\}$ endowed with the Lorentzian metric obtained by restriction of the quadratic form $\mathrm{q}_{2, n}$ is called anti-de Sitter space $\operatorname{AdS}_{n+1}$.

Observe the analogy with the definition of the hyperbolic space $\mathbb{H}^{n}$. Considering the coordinates $\left(r, \theta, x_{1}, \ldots, x_{n}\right)$, where $u=r \cos (\theta)$ and $v=r \sin (\theta)$, it turns out that for every real number $\theta_{0}$ the subset

$$
H_{\theta_{0}}:=\left\{\left(r, \theta, x_{1}, \ldots, x_{n}\right) \mid \theta=\theta_{0}\right\} \subset \mathbb{R}^{2, n}
$$

is a totally geodesic copy of the hyperbolic space embedded in $\operatorname{AdS}_{n+1}$. More generally, the totally geodesic subspaces of dimension $k$ in $\mathrm{AdS}_{n+1}$ are connected components of the intersections of $\mathrm{AdS}_{n+1}$ with the linear subspaces of dimension $(k+1)$ in $\mathbb{R}^{2, n}$. As a particular case, the geodesics of $\operatorname{AdS}_{n+1}$ are obtained by intersecting it with 2-planes.

Remark 2.2. We will often need an auxiliary Euclidean metric on $\mathbb{R}^{2, n}$. Let us fix once for all the Euclidean norm $\|\cdot\|_{0}$ defined by

$$
\left\|\left(u, v, x_{1}, \ldots, x_{n}\right)\right\|_{0}^{2}:=u^{2}+v^{2}+x_{1}^{2}+\cdots+x_{n}^{2} .
$$

\subsection{Conformal model}

Proposition 2.3. The anti-de Sitter space $\mathrm{AdS}_{n+1}$ is conformally equivalent to $\left(\mathbb{S}^{1} \times \mathbb{D}^{n},-d \theta^{2}+d s^{2}\right)$, where $d \theta^{2}$ is the standard Riemannian metric on $\mathbb{S}^{1}=$ $\mathbb{R} / 2 \pi \mathbb{Z}$ and $d s^{2}$ is the standard metric (of constant curvature +1 ) on the sphere $\mathbb{S}^{n}$ and $\mathbb{D}^{n}$ is the open upper hemisphere of $\mathbb{S}^{n}$.

Proof. In the $\left(r, \theta, x_{1}, \ldots, x_{n}\right)$-coordinates the AdS metric is

$$
-r^{2} \mathrm{~d} \theta^{2}+\mathrm{ds}_{\text {hyp }}^{2}
$$

where $\mathrm{ds}_{\text {hyp }}^{2}$ denotes the hyperbolic metric, that is, the induced metric on $H_{0}=$ $\left\{\left(r, \theta, x_{1}, \ldots, x_{n}\right) \mid \theta=0\right\} \approx \mathbb{H}^{n}$. More precisely, $H_{0}$ is a sheet of the hyperboloid $\left\{\left(r, x_{1}, \ldots, x_{n}\right) \in \mathbb{R}^{1, n} \mid-r^{2}+x_{1}^{2}+\cdots+x_{n}^{2}=-1\right\}$. The map $\left(r, x_{1}, \ldots, x_{n}\right) \rightarrow$ $\left(1 / r, x_{1} / r, \ldots, x_{n} / r\right)$ sends this hyperboloid to $\mathbb{D}^{n}$, and an easy computation shows that the pull-back of the standard metric on the hemisphere by this map is $r^{-2} \mathrm{ds}_{\text {hyp }}^{2}$. The proposition follows.

Proposition 2.3 shows in particular that $\operatorname{AdS}_{n+1}$ contains many closed causal curves. But the universal covering $\widetilde{\mathrm{AdS}}_{n+1}$, which is conformally equivalent to $\left(\mathbb{R} \times \mathbb{D}^{n},-d \theta^{2}+d s^{2}\right)$, contains no periodic causal curve. It turns out to be strongly causal but not globally hyperbolic. 
2.3. Einstein universe. We define the Einstein universe $\operatorname{Ein}_{n+1}$ as the product $\mathbb{S}^{1} \times$ $\mathbb{S}^{n}$ endowed with the metric $-d \theta^{2}+d s^{2}$ where $d s^{2}$ is, as above, the standard spherical metric. The universal Einstein universe $\widetilde{\text { Ein }}_{n+1}$ is the cyclic covering $\mathbb{R} \times \mathbb{S}^{n}$ equipped with the lifted metric which we still denote by $-d \theta^{2}+d s^{2}$, but where $\theta$ now takes value in the real line. According to this definition, the Einstein spaces $\operatorname{Ein}_{n+1}$ and $\widetilde{\operatorname{Ein}}_{n+1}$ are Lorentzian manifolds, but it is more adequate to consider them as conformal Lorentzian manifolds. We fix a time orientation: the one for which the coordinate $\theta$ is a time function on $\widetilde{\operatorname{Ein}}_{n+1}$.

In the sequel, we denote by $\mathrm{p}: \widetilde{\operatorname{Ein}}_{n+1} \rightarrow \operatorname{Ein}_{n+1}$ the cyclic covering map. Let $\delta: \widetilde{\operatorname{Ein}}_{n+1} \rightarrow \widetilde{\operatorname{Ein}}_{n+1}$ be a generator of the Galois group of this cyclic covering such that for any $\tilde{x}$ in $\widetilde{\operatorname{Ein}}_{n+1}$ the image $\delta(\tilde{x})$ is in the future of $\tilde{x}$.

Even if the Einstein universe is merely a conformal Lorentzian spacetime, the notion of photon, i.e., (non-parameterized) light-like geodesics, is well defined. This allows to associate to each point its lightcone $C(x)$, defined as the union of photons containing $x$. If the point $x$ is given by a pair $(\theta, \mathrm{x})$ in $\mathbb{S}^{1} \times \mathbb{S}^{n}$, the lightcone $C(x)$ is the set of pairs $\left(\theta^{\prime}, \mathrm{y}\right)$ such that $\left|\theta^{\prime}-\theta\right|=d(\mathrm{x}, \mathrm{y})$ where $d$ is distance function for the spherical metric $d s^{2}$.

There is only one point in $\mathbb{S}^{n}$ at distance $\pi$ to $\mathrm{x}$ : the antipodal point $-\mathrm{x}$. This points admit a single lifting in $\operatorname{Ein}_{n+1}$ that belongs to $C(x)$ : we will also call it the antipodal point, and denote it by $-x=(\theta+\pi,-\mathrm{x})$. Removing the point $x$ and its antipodal $-x$ from the lightcone $C(x)$ separates it in two connected components: the future cone $C^{+}(x)$ and the past cone $C^{-}(x)$. One has:

$$
\begin{aligned}
& C^{+}(x)=\left\{\left(\theta^{\prime}, \mathrm{y}\right) \mid \theta<\theta^{\prime}<\theta+\pi, d(\mathrm{x}, \mathrm{y})=\theta^{\prime}-\theta\right\}, \\
& C^{-}(x)=\left\{\left(\theta^{\prime}, \mathrm{y}\right) \mid \theta-\pi<\theta^{\prime}<\theta, d(\mathrm{x}, \mathrm{y})=\theta-\theta^{\prime}\right\} .
\end{aligned}
$$

Observe that the future cone of $x$ is the past cone of $-x$, and that, conversely, the past cone of $x$ is the future cone of $-x$.

According to Proposition 2.3 the anti de Sitter space $\operatorname{AdS}_{n+1}$ (resp. $\widetilde{\mathrm{AdS}}_{n+1}$ ) conformally embeds into the Einstein space $\operatorname{Ein}_{n+1}\left(\right.$ resp. $\left.\widetilde{\operatorname{Ein}}_{n+1}\right)$. Hence the time orientation on $\operatorname{Ein}_{n+1}$ selected above induces a time orientation on $\operatorname{AdS}_{n+1}$ and $\widetilde{\operatorname{AdS}}_{n+1}$. Since the boundary $\partial \mathbb{D}^{n}$ is an equatorial sphere, the boundary $\partial \widetilde{\operatorname{AdS}}_{n+1}$ is a copy of the Einstein universe $\widetilde{\operatorname{Ein}}_{n}$. In other words, one can attach a "Penrose boundary" $\partial \widetilde{\operatorname{AdS}}_{n+1}$ to $\widetilde{\mathrm{AdS}}_{n+1}$ such that $\widetilde{\operatorname{AdS}}_{n+1} \cup \partial \widetilde{\mathrm{AdS}}_{n+1}$ is conformally equivalent to $\left(\mathbb{S}^{1} \times \overline{\mathbb{D}}^{n},-d \theta^{2}+d s^{2}\right)$, where $\overline{\mathbb{D}}^{n}$ is the closed upper hemisphere of $\mathbb{S}^{n}$.

The restrictions of the covering map $\mathrm{p}$ and the generator $\delta$ to $\widetilde{\mathrm{AdS}}_{n+1} \subset \widetilde{\operatorname{Ein}}_{n+1}$ are respectively a covering map over $\operatorname{AdS}_{n+1}$ and a generator of the Galois group of the covering; we will still denote them by $\mathrm{p}$ and $\delta$.

2.4. Isometry groups. Every element of $\mathrm{SO}(2, n)$ induces an isometry of $\operatorname{AdS}_{n+1}$, and, for $n \geq 2$, every isometry of $\operatorname{AdS}_{n+1}$ comes from an element of $\operatorname{SO}(2, n)$. Similarly, conformal isometries of $\operatorname{Ein}_{n+1}$ are projections of elements of $\mathrm{SO}(2, n+1)$ acting on $\ell_{n+1}$ (still for $n \geq 2$ ). 
In the sequel, we will only consider isometries preserving the orientation and the time orientation, i.e., elements of the neutral component $\mathrm{SO}_{0}(2, n)\left(\right.$ or $\left.\mathrm{SO}_{0}(2, n+1)\right)$.

2.5. Achronal subsets. Recall that a subset of a conformal Lorentzian manifold is achronal (respectively acausal) if there is no time-like (respectively causal) curve joining two distinct points of the subset. In $\operatorname{Ein}_{n} \approx\left(\mathbb{R} \times \mathbb{S}^{n-1},-d \theta^{2}+d s^{2}\right)$, every achronal subset is precisely the graph of a 1-Lipschitz function $f: \Lambda_{0} \rightarrow \mathbb{R}$, where $\Lambda_{0}$ is a subset of $\mathbb{S}^{n-1}$ endowed with its canonical metric $d$. In particular, the achronal closed topological hypersurfaces in $\partial \widetilde{\mathrm{AdS}}_{n+1}$ can be obtained as graphs of 1-Lipschitz functions $f: \mathbb{S}^{n-1} \rightarrow \mathbb{R}$. In particular, they are topological $(n-1)$-spheres.

Similarly, achronal subsets of $\widetilde{\mathrm{AdS}}_{n+1}$ are graphs of 1-Lipschitz functions $f: \Lambda_{0} \rightarrow \mathbb{R}$, where $\Lambda_{0}$ is a subset of $\mathbb{D}^{n}$, and achronal topological hypersurfaces are graphs of 1-Lipschitz maps $f: \mathbb{D}^{n} \rightarrow \mathbb{R}$.

Stricto sensu there is no achronal subset in $\operatorname{Ein}_{n+1}$ since closed time-like curves through a given point cover the entire $\operatorname{Ein}_{n+1}$. Nevertheless, we can keep track of this notion in $\operatorname{Ein}_{n+1}$ by defining "achronal" subsets of $\operatorname{Ein}_{n+1}$ as projections of genuine achronal subsets of $\widetilde{\operatorname{Ein}}_{n+1}$. This definition is justified by the following results:

Lemma 2.4. The restriction of $\mathrm{p}$ to any achronal subset of $\widetilde{\mathrm{Ein}}_{n+1}$ is injective.

Proof. Since the diameter of $\mathbb{S}^{n}$ is $\pi$, the difference between the $t$-coordinates of two elements of an achronal subset of $\widetilde{\operatorname{Ein}}_{n+1}$ is at most $\pi$. The lemma follows immediately.

Corollary 2.5. Let $\widetilde{\Lambda}_{1}, \widetilde{\Lambda}_{2}$ be two achronal subsets of $\widetilde{\operatorname{Ein}}_{n+1}$ admitting the same projection in $\operatorname{Ein}_{n+1}$. Then there is an integer $k$ such that

$$
\tilde{\Lambda}_{1}=\delta^{k} \tilde{\Lambda}_{2}
$$

where $\delta$ is the generator of the Galois group introduced above.

2.6. The Klein model $\mathbb{A D S}_{\boldsymbol{n}+\mathbf{1}}$ of the anti-de Sitter space. We now consider the quotient $\mathbb{S}\left(\mathbb{R}^{2, n}\right)$ of $\mathbb{R}^{2, n} \backslash\{0\}$ by positive homotheties. In other words, $\mathbb{S}\left(\mathbb{R}^{2, n}\right)$ is the double covering of the projective space $\mathbb{P}\left(\mathbb{R}^{2, n}\right)$. We denote by $\mathbb{S}$ the projection function from $\mathbb{R}^{2, n} \backslash\{0\}$ to $\mathbb{S}\left(\mathbb{R}^{2, n}\right)$. Note that its restriction to $\operatorname{AdS}_{n+1}$ is one-to-one. We define the Klein model $\mathbb{A D S}_{n+1}$ of the anti-de Sitter space as the projection of $\operatorname{AdS}_{n+1}$ in $\mathbb{S}\left(\mathbb{R}^{2, n}\right)$, endowed with the induced Lorentzian metric.

$A \mathbb{D S} S_{n+1}$ is also the projection of the open domain of $\mathbb{R}^{2, n}$ defined by the inequality $\left\{\mathrm{q}_{2, n}<0\right\}$. The topological boundary of $\mathbb{A D S} \mathbb{S}_{n+1}$ in $\mathbb{S}\left(\mathbb{R}^{2, n}\right)$ is the projection of the isotropic cone $\ell_{n}$; we will denote this boundary by $\partial \mathbb{A D S}_{n+1}$. By construction, the projection $\mathbb{S}$ defines an isometry between $\operatorname{AdS}_{n+1}$ and $\mathbb{A D S} \mathbb{S}_{n+1}$. The continuous extension of this isometry provides a canonical homeomorphism between $\operatorname{AdS}_{n+1} \cup \partial \mathrm{AdS}_{n+1}$ and $\mathbb{A D} \mathbb{S}_{n+1} \cup \partial \mathbb{A D} \mathbb{S}_{n+1}$. 
For every linear subspace $F$ of dimension $k+1$ in $\mathbb{R}^{2, n}$, we denote by $\mathbb{S}(F):=$ $\mathbb{S}(F \backslash\{0\})$ the corresponding projective subspace of dimension $k$ in $\mathbb{S}\left(\mathbb{R}^{2, n}\right)$. The geodesics of $\mathbb{A D S} \mathbb{S}_{n+1}$ are the connected components of the intersections of $\mathbb{A D} \mathbb{S}_{n+1}$ with the projective lines $\mathbb{S}(F)$ of $\mathbb{S}\left(\mathbb{R}^{2, n}\right)$. More generally, the totally geodesic subspaces of dimension $k$ in $\mathbb{A D S} S_{n+1}$ are the connected components of the intersections of $\mathbb{A D S} \mathbb{S}_{n+1}$ with the projective subspaces $\mathbb{S}(F)$ of dimension $k$ of $\mathbb{S}\left(\mathbb{R}^{2, n}\right)$.

Definition 2.6. For every $x$ in $\operatorname{AdS}_{n+1}$, the affine domain $U(x)$ of $\mathbb{A D} \mathbb{S}_{n+1}$ is the connected component of $\mathbb{A D} \mathbb{S}_{n+1} \backslash \mathbb{S}\left(x^{\perp}\right)$ containing $x$. Let $V(x)$ be the connected component of $\mathbb{S}\left(\mathbb{R}^{2, n}\right) \backslash \mathbb{S}\left(x^{\perp}\right)$ containing $U(x)$. The boundary $\partial U(x) \subset \partial \mathbb{A D} \mathbb{S}_{n+1}$ of $U(x)$ in $V(x)$ is called the affine boundary of $U(x)$.

Remark 2.7. Up to composition by an element of the isometry group $\mathrm{SO}_{0}(2, n)$ of $\mathrm{q}_{2, n}$, we can assume that $\mathbb{S}\left(x^{\perp}\right)$ is the projection of the hyperplane $\{u=0\}$ in $\mathbb{R}^{2, n}$ and $V(x)$ is the projection of the region $\{u>0\}$ in $\mathbb{R}^{2, n}$. The map

$$
\left(u, v, x_{1}, x_{2}, \ldots, x_{n+1}\right) \mapsto\left(t, \bar{x}_{1}, \ldots, \bar{x}_{n}\right):=\left(\frac{v}{u}, \frac{x_{1}}{u}, \frac{x_{2}}{u}, \ldots, \frac{x_{n}}{u}\right)
$$

induces a diffeomorphism between $V(x)$ and $\mathbb{R}^{n+1}$ mapping the affine domain $U(x)$ to the region $\left\{\left(t, \bar{x}_{1}, \ldots, \bar{x}_{n}\right) \in \mathbb{R}^{n+1} \mid \mathrm{q}_{1, n}\left(t, \bar{x}_{1}, \ldots, \bar{x}_{n}\right)<1\right\}$, where $\mathrm{q}_{1, n}$ is the Minkowski norm. The affine boundary $\partial U(x)$ corresponds to the hyperboloid $\left\{\left(t, \bar{x}_{1}, \ldots, \bar{x}_{n} \mid \mathrm{q}_{1, n}\left(t, \bar{x}_{1}, \ldots, \bar{x}_{n}\right)=1\right\}\right.$. The intersections between $U(x)$ and the totally geodesic subspaces of $\mathbb{A D} \mathbb{S}_{n+1}$ correspond to the intersections of the region $\left\{\left(t, \bar{x}_{1}, \ldots, \bar{x}_{n}\right) \in \mathbb{R}^{n+1} \mid \mathrm{q}_{1, n}\left(t, \bar{x}_{1}, \ldots, \bar{x}_{n}\right)<1\right\}$ with the affine subspaces of $\mathbb{R}^{n+1}$.

Although the real number $\langle x \mid y\rangle$ is well defined only for $x, y \in \mathbb{R}^{2, n}$, its sign is well defined for $x, y \in \mathbb{S}\left(\mathbb{R}^{2, n}\right)$ :

Lemma 2.8. Let $U$ be an affine domain in $\mathbb{A D S}_{n+1}$ and $\partial U \subset \partial \mathbb{A D} \mathbb{S}_{n+1}$ be its affine boundary. Let $x$ be a point in $\partial U$ and let $y$ be a point in $U \cup \partial U$. There exists a causal (resp. time-like) curve joining $x$ to $y$ in $U \cup \partial U$ if and only if $\langle x \mid y\rangle \geq 0$ (resp. $\langle x \mid y\rangle>0)$.

Proof. See e.g. [Bar08b], Proposition 5.10, or [BBZ07], Proposition 4.19.

2.7. The Klein model of the Einstein universe. The Einstein universe also has a Klein model, obtained by projecting the isotropic cone $\ell_{n}$ of $\mathbb{R}^{2, n}$ to $\mathbb{S}\left(\mathbb{R}^{2, n}\right)$. The conformal Lorentzian structure can then be defined in terms of the quadratic form $\mathrm{q}_{2, n}$. In particular, an immediate corollary of Lemma 2.8 is:

Corollary 2.9. For $\Lambda \subseteq \operatorname{Ein}_{n}$, the following assertions are equivalent.

(1) $\Lambda$ is achronal (respectively acausal); 
(2) when we view $\Lambda$ as a subset of $\mathbb{S}\left(\mathcal{C}_{n}\right) \approx \operatorname{Ein}_{n}$, the scalar product $\langle x \mid y\rangle$ is non-positive (respectively negative) for every distinct $x, y \in \Lambda$.

In the sequel, we will frequently need to change from one model to the other. In order to simplify things, we will often identify $\operatorname{Ein}_{n}$ with $\mathbb{S}\left(\mathcal{C}_{n}\right)$. More details about the Einstein universe can be found in [Fra05], [BCD $\left.{ }^{+} 08\right]$.

Remark 2.10. The affine boundary $\partial U(x)$ defined in Remark 2.7 as a domain of $\operatorname{Ein}_{n}$ is conformally isometric to the de Sitter space. Hence we also call it de Sitter domain.

2.8. Unit tangent bundle. Denote by $\mathcal{E}^{1} \mathrm{AdS}_{n+1}$ (resp. $\mathscr{L}^{1} \mathrm{AdS} \mathrm{S}_{n+1}$ ) the tangent bundle consisting of unit space-like (resp. light-like) tangent vectors. For any spacelike or light-like tangent vector $(x, v)$ in $\operatorname{AdS}_{n+1}$, the geodesic issued from the point $x$ with speed $v$ has a future and past limit in the Einstein universe. We denote by $\ell^{ \pm}: \mathcal{E}^{1} \mathrm{AdS}_{n+1} \cup \mathscr{L}^{1} \mathrm{AdS}_{n+1} \rightarrow \operatorname{Ein}_{n}$ the mapping which assigns such a vector to its limit ( $\ell^{-}$for the past limit, $\ell^{+}$for the future limit).

\section{Regular AdS manifolds}

3.1. AdS regular domains. Let $\tilde{\Lambda}$ be a closed achronal subset of $\partial \widetilde{\mathrm{AdS}}_{n+1}$, and $\Lambda$ be the projection of $\widetilde{\Lambda}$ in $\partial \operatorname{AdS}_{n+1}$. We denote by $\widetilde{E}(\widetilde{\Lambda})$ the invisible domain of $\widetilde{\Lambda}$ in $\widetilde{\operatorname{AdS}}_{n+1} \cup \partial \widetilde{\mathrm{AdS}}_{n+1}$, that is,

$$
\widetilde{E}(\tilde{\Lambda})=:\left(\widetilde{\operatorname{AdS}}_{n+1} \cup \partial \widetilde{\operatorname{AdS}}_{n+1}\right) \backslash\left(J^{-}(\tilde{\Lambda}) \cup J^{+}(\tilde{\Lambda})\right),
$$

where $J^{-}(\tilde{\Lambda})$ and $J^{+}(\tilde{\Lambda})$ are the causal past and the causal future of $\widetilde{\Lambda}$ in $\widetilde{\operatorname{AdS}}_{n+1} \cup$ $\partial \widetilde{\operatorname{AdS}}_{n+1}=\left(\mathbb{R} \times \overline{\mathbb{D}}^{n-1},-d \theta^{2}+d s^{2}\right)$. We denote by $\mathrm{Cl}(\widetilde{E}(\tilde{\Lambda}))$ the closure of $\widetilde{E}(\widetilde{\Lambda})$ in $\widetilde{\operatorname{AdS}}_{n+1} \cup \partial \widetilde{\operatorname{AdS}}_{n+1}$ and by $E(\Lambda)$ the projection of $\widetilde{E}(\tilde{\Lambda})$ in $\operatorname{AdS}_{n+1} \cup \partial \operatorname{AdS}_{n+1}$ (according to Corollary $2.5, E(\Lambda)$ only depends on $\Lambda$, not on $\widetilde{\Lambda}$ ).

Definition 3.1. A $n$-dimensional $A d S$ regular domain is a domain of the form $E(\Lambda)$ where $\Lambda$ is the projection in $\partial \operatorname{AdS}_{n+1}$ of an achronal subset $\widetilde{\Lambda} \subset \partial \widetilde{\operatorname{AdS}}_{n+1}$ containing at least two points. If $\widetilde{\Lambda}$ is a topological $(n-1)$-sphere, then $E(\Lambda)$ is said to be $G H$ regular. This definition is motivated by Theorem 4.3 and Proposition 4.5.

Remark 3.2. For every closed achronal set $\widetilde{\Lambda}$ in $\partial \widetilde{\operatorname{AdS}}_{n+1}$, the invisible domain $\widetilde{E}(\widetilde{\Lambda})$ is causally convex in $\widetilde{\mathrm{AdS}}_{n+1} \cup \partial \widetilde{\mathrm{AdS}}_{n+1}$ : this is an immediate consequence of the definitions. It follows that AdS regular domains are strongly causal.

Definition 3.3. Let $\widetilde{\Lambda}$ be a closed achronal subset of $\partial \widetilde{\mathrm{AdS}}_{n+1}$. Recall from $\S 2.5$ that $\tilde{\Lambda}$ is the graph of a 1-Lipschitz function $f: \Lambda_{0} \rightarrow \mathbb{R}$, where $\Lambda_{0}$ is a closed subset of 
$\mathbb{S}^{n-1}$. Define two functions $f^{-}, f^{+}: \overline{\mathbb{D}}^{n} \rightarrow \mathbb{R}$ as follows:

$$
\begin{aligned}
& f^{-}(\mathrm{x}):=\sup _{\mathrm{y} \in \Lambda_{0}}\{f(\mathrm{y})-d(\mathrm{x}, \mathrm{y})\}, \\
& f^{+}(\mathrm{x}):=\inf _{\mathrm{y} \in \Lambda_{0}}\{f(\mathrm{y})+d(\mathrm{x}, \mathrm{y})\},
\end{aligned}
$$

where $d$ is the distance induced by $\mathrm{d} s^{2}$ on $\overline{\mathbb{D}}^{n}$. It is easy to check that

$$
\widetilde{E}(\tilde{\Lambda})=\left\{(\theta, \mathrm{x}) \in \mathbb{R} \times \overline{\mathbb{D}}^{n} \mid f^{-}(\mathrm{x})<\theta<f^{+}(\mathrm{x})\right\} .
$$

The following lemma is a refinement of Lemma 2.4:

Lemma 3.4. For every (non-empty) closed achronal set $\widetilde{\Lambda} \subset \partial \widetilde{\mathrm{AdS}}_{n+1}$, the projection of $\widetilde{E}(\widetilde{\Lambda})$ on $E(\Lambda)$ is one-to-one.

Proof. We use the notations introduced in the Definition 3.3. For every $\mathrm{x} \in \overline{\mathbb{D}}^{n}$, there exists a point $\mathrm{y}$ in the sphere $\mathbb{S}^{n-1}=\partial \overline{\mathbb{D}}^{n}$ such that $d(\mathrm{x}, \mathrm{y}) \leq \pi / 2$. Hence, for every $\mathrm{x} \in \overline{\mathbb{D}}^{n}$, we have $f^{+}(\mathrm{x})-f^{-}(\mathrm{x}) \leq \pi$. This means that $\widetilde{E}(\tilde{\Lambda})$ lies in $E=\left\{(\theta, \mathrm{x}) \in \mathbb{R} \times \overline{\mathbb{D}}^{n} \mid f^{-}(\mathrm{x})<\theta<f^{-}(\mathrm{x})+\pi\right\}$. The restriction to this set $E$ of the projection function from $\widehat{\operatorname{AdS}}_{n+1} \cup \partial \widetilde{\mathrm{AdS}}_{n+1}=\mathbb{R} \times \overline{\mathbb{D}}^{n}$ to $\mathrm{AdS}_{n+1} \cup \partial \operatorname{AdS}_{n+1}=$ $(\mathbb{R} / 2 \pi \mathbb{Z}) \times \overline{\mathbb{D}}^{n-1}$ is obviously one-to-one.

Definition 3.5. An achronal subset $\widetilde{\Lambda}$ of $\widetilde{\operatorname{Ein}}_{n+1}$ is called purely light-like if the associated subset $\Lambda_{0}$ of $\mathbb{S}^{n}$ contains two antipodal points $\mathrm{x}_{0}$ and $-\mathrm{x}_{0}$ such that, for the corresponding 1-Lipschitz map $f: \Lambda_{0} \rightarrow \mathbb{R}$, the equality $f\left(\mathrm{x}_{0}\right)=f\left(-\mathrm{x}_{0}\right)+\pi$ holds.

If $\tilde{\Lambda}$ is purely light-like, for every element $\mathrm{x}$ of $\overline{\mathbb{D}}^{n}$ we have $f^{-}(\mathrm{x})=f^{+}(\mathrm{x})=$ $f\left(-\mathrm{x}_{0}\right)+d\left(-\mathrm{x}_{0}, \mathrm{x}\right)=f\left(\mathrm{x}_{0}\right)-d\left(\mathrm{x}_{0}, \mathrm{x}\right)$, implying that $\widetilde{E}(\widetilde{\Lambda})$ is empty. Conversely, we have:

Lemma 3.6. $\tilde{E}(\tilde{\Lambda})$ is empty if and only if $\tilde{\Lambda}$ is purely light-like. More precisely, if for some point $\mathrm{x}$ in $\mathbb{D}^{n}$ the equality $f^{+}(\mathrm{x})=f^{-}(\mathrm{x})$ holds then $\tilde{\Lambda}$ is purely light-like.

Proof. Assume $f^{+}(\mathrm{x})=f^{-}(\mathrm{x})$ for some $\mathrm{x}$ in $\mathbb{D}^{n}$. By compactness of $\Lambda_{0}$, the upper and lower bounds in the definitions of $f^{ \pm}$are attained, i.e., there are $\mathrm{y}^{ \pm}$in $\Lambda_{0}$ such that

$$
f^{-}(\mathrm{x})=f\left(\mathrm{y}^{-}\right)-d\left(\mathrm{x}, \mathrm{y}^{-}\right), \quad f^{+}(\mathrm{x})=f\left(\mathrm{y}^{+}\right)+d\left(\mathrm{x}, \mathrm{y}^{+}\right) .
$$

As a consequence,

$$
d\left(\mathrm{y}^{-}, \mathrm{y}^{+}\right) \geq f\left(\mathrm{y}^{-}\right)-f\left(\mathrm{y}^{+}\right)=d\left(\mathrm{y}^{-}, \mathrm{x}\right)+d\left(\mathrm{x}, \mathrm{y}^{+}\right) .
$$

We are in the equality case of the triangular inequality. It follows that $x$ belongs to a minimizing geodesic in $\mathbb{S}^{n}$ joining $\mathrm{y}^{-}$to $\mathrm{y}^{+}$. This is possible only if the points 
$\mathrm{y}^{+}, \mathrm{y}^{-}$are antipodal to each other - if not, the minimizing geodesic joining would be unique and contained in $\partial \mathbb{D}^{n}$. Moreover, $f\left(\mathrm{y}^{-}\right)=f\left(\mathrm{y}^{+}\right)+\pi$. The lemma follows.

Corollary 3.7. For every achronal topological $(n-1)$-sphere $\tilde{\Lambda} \subset \partial \widetilde{\mathrm{AdS}}_{n+1}$,

(1) $\widetilde{E}(\widetilde{\Lambda})$ is disjoint from $\partial \widetilde{\mathrm{AdS}}_{n+1}$ (i.e., it is contained in $\left.\widetilde{\mathrm{AdS}}_{n+1}\right)$;

(2) $\operatorname{Cl}(\widetilde{E}(\widetilde{\Lambda})) \cap \partial \widetilde{\mathrm{AdS}}_{n+1}=\widetilde{\Lambda}$, where $C l(\widetilde{E}(\tilde{\Lambda}))$ denotes the closure of $\widetilde{E}(\widetilde{\Lambda})$ in $\widetilde{\operatorname{Ein}}_{n+1}$.

Proof. We use the notations introduced in the Definition 3.3. Since $\widetilde{\Lambda}$ is a topological $(n-1)$-sphere, the set $\Lambda_{0}$ is the whole sphere $\mathbb{S}^{n-1}$. For every $\mathrm{x} \in \mathbb{S}^{n-1}=\Lambda_{0}$, one has $f^{-}(\mathrm{x})=f^{+}(\mathrm{x})=f(\mathrm{x})$. Finally, recall that $(\theta, \mathrm{x}) \in \widetilde{E}(\widetilde{\Lambda})($ resp. $(\theta, \mathrm{x}) \in$ $\mathrm{Cl}(\widetilde{E}(\widetilde{\Lambda})))$ if and only if $f^{-}(\mathrm{x})<\theta<f^{+}(\mathrm{x})\left(\right.$ resp. $\left.f^{-}(\mathrm{x}) \leq \theta \leq f^{+}(\mathrm{x})\right)$. The corollary follows.

Remark 3.8. It follows from item (2) of Corollary 3.7 that the GH-regular domain $E(\Lambda)$ characterizes $\Lambda$, i.e., invisible domains of different achronal $(n-1)$-spheres are different. We call $\Lambda$ the limit set of $E(\Lambda)$.

3.2. AdS regular domains as subsets of $\mathbb{A D S}_{n+1}$. The canonical homeomorphism between $\mathrm{AdS}_{n+1} \cup \partial \mathrm{AdS}_{n+1}$ and $\mathbb{A D} \mathbb{S}_{n+1} \cup \partial \mathbb{A D} \mathbb{S}_{n+1}$ allows us to see AdS regular domains as subsets of $\mathbb{A D S} S_{n+1}$.

Lemma 3.9. Let $\Lambda \subset \partial \mathrm{AdS}_{n+1}$ be the projection of a closed achronal subset of $\partial \widetilde{\mathrm{AdS}}_{n+1}$ which is not purely light-like. We see $\Lambda$ and $E(\Lambda)$ in $\mathbb{A D} \mathbb{S}_{n+1} \cup \partial \mathbb{A D} \mathbb{S}_{n+1}$. Then $\Lambda$ and $E(\Lambda)$ are contained in the union $U \cup \partial U$ of an affine domain and its affine boundary.

Proof. See [Bar08b], Lemma 8.27.

Lemma 3.9 implies, in particular, that every AdS regular domain is contained in an affine domain of $\mathbb{A D S}_{n+1}$. This allows to visualize AdS regular domains as subsets of $\mathbb{R}^{n+1}$ (see Remark 2.7).

Putting together the definition of the invisible domain $E(\Lambda)$ of a set $\Lambda \subset \partial \operatorname{AdS}_{n+1}$ and Lemma 2.8, one gets:

Proposition 3.10. Let $\Lambda \subset \partial \mathrm{AdS}_{n+1}$ be the projection of a closed achronal subset of $\partial \widetilde{\mathrm{AdS}}_{n+1}$ which is not purely light-like. If we see $\Lambda$ and $E(\Lambda)$ in the Klein model $\mathbb{A D} \mathbb{S}_{n+1} \cup \partial \mathbb{A D} \mathbb{S}_{n+1}$, then

$$
\left.E(\Lambda)=\left\{y \in \mathbb{A D S}_{n+1} \cup \partial \mathbb{A D} \mathbb{S}_{n+1} \text { such that }\langle y \mid x\rangle<0 \text { for every } x \in \Lambda\right\}\right) .
$$

Remark 3.11. A nice (and important) corollary of this proposition is that the invisible domain $E(\Lambda)$ associated with a set $\Lambda$ is always geodesically convex: any geodesic joining two points in $E(\Lambda)$ is contained in $E(\Lambda)$. 
3.3. Convex hull of AdS regular domains. According to Lemma 3.9 the limit set $\Lambda$ and the regular domain $E(\Lambda)$ are contained in $U \cup \partial U$ where $U$ is an affine domain of $\operatorname{AdS}_{n+1}$. In particular, it is contained in an affine chart $V$ of $\mathbb{S}\left(\mathbb{R}^{2, n}\right)$. We can consider the convex hull $\operatorname{Conv}(\Lambda)$ of $\Lambda$ in this affine chart. This convex hull does not depend on the choice of $V$. Moreover, since $E(\Lambda)$ is convex, it contains $\operatorname{Conv}(\Lambda)$ (cf. Remark 3.11). For more details, see for example [Bar08b].

Alternatively, we also can define $\operatorname{Conv}(\Lambda)$ as the projection $\mathbb{S}(C)$ where $C$ is the set of barycentric combinations $t_{1} x_{1}+\cdots+t_{k} x_{k}$ where $t_{i}$ are non-negative real numbers such that $t_{1}+\cdots+t_{k}=1$ and $x_{i}$ elements of $\ell_{n} \subset \mathbb{R}^{2, n}$ the projections $\mathbb{S}\left(x_{i}\right)$ of which belong to $\Lambda$.

Lemma 3.12. The convex hull $\operatorname{Conv}(\Lambda)$ is compact. Its intersection with $\partial \mathrm{AdS}_{n+1}$ is $\Lambda$, and the "finite part" $\operatorname{Conv}(\Lambda) \cap \operatorname{AdS}_{n+1}=\operatorname{Conv}(\Lambda) \backslash \Lambda$ is contained in $E(\Lambda)$.

Proof. The compactness of $\operatorname{Conv}(\Lambda)$ follows from the compactness of $\Lambda$. Let $x=$ $t_{1} x_{1}+\cdots+t_{k} x_{k}$ be an element of $\mathbb{R}^{2, n}$ projecting in $\mathbb{S}\left(\mathbb{R}^{2, n}\right)$ on an element of $\operatorname{Conv}(\Lambda)$. For every $y$ such that $\mathbb{S}(y)$ belongs to $\Lambda$, according to Corollary 2.9,

$$
\langle x \mid y\rangle=\sum_{i=1}^{k} t_{i}\left\langle x_{i} \mid y\right\rangle \leq 0 .
$$

Moreover, if $\langle x \mid y\rangle$ vanishes, then every $\left\langle y \mid x_{i}\right\rangle$ vanishes. But since $\Lambda$ is acausal, $\left\langle y \mid x_{i}\right\rangle=0$ implies $y=x_{i}$ : according to Proposition 3.10 $\operatorname{Conv}(\Lambda) \backslash \Lambda$ is contained in $E(\Lambda)$. The lemma follows since $E(\Lambda)$ is contained in $\operatorname{AdS}_{n+1}$.

Lemma 3.13. If $\operatorname{Conv}(\Lambda) \cap \operatorname{AdS}_{n+1}$ has empty interior, then it is contained in a unique totally geodesic space-like hypersurface of $\mathrm{AdS}_{n+1}$.

Proof. If $\operatorname{Conv}(\Lambda)$ has empty interior, it is contained in a projective hyperplane $\mathbb{S}\left(v^{\perp}\right)$. If $\mathrm{q}_{2, n}(v)$ is positive, then $\mathbb{S}\left(v^{\perp}\right) \cap \operatorname{AdS}_{n+1}$ is an isometric, totally geodesic embedding of $\operatorname{AdS}_{n}$ which, in a well-chosen conformal chart $\operatorname{AdS}_{n+1} \approx \mathbb{S}^{1} \times \mathbb{D}^{n}$, corresponds to $\left\{x_{n}=0\right\}$. The set $\Lambda$, which is a graph over $\partial \mathbb{D}^{n}$, cannot be contained in the closure of $\left\{x_{n}=0\right\}$, thus yielding a contradiction. Similarly, from the assumption $\mathrm{q}_{2, n}(v)=0$ one would be able to deduce that $\Lambda$ is purely light-like.

Hence, up to renormalization, $v$ lies in $\operatorname{AdS}_{n+1}$, and $\operatorname{Conv}(\Lambda) \cap \operatorname{AdS}_{n+1}$ is contained in the totally geodesic hypersurface $\mathbb{S}\left(v^{\perp}\right) \cap \operatorname{AdS}_{n+1}$. This convex set cannot be contained in a different totally geodesic hypersurface $\mathbb{S}\left(\left(v^{\prime}\right)^{\perp}\right) \cap \operatorname{AdS}_{n+1}$ $\left(v \neq v^{\prime}\right)$ because the dimension of the intersection of $\mathbb{S}\left(\left(v^{\prime}\right)^{\perp}\right) \cap \mathbb{S}\left(\left(v^{\prime}\right)^{\perp}\right)$ with the Einstein space is $n-2$ dimensional, thus forbidding the inclusion of a topological $(n-1)$-sphere $\Lambda$.

For the remaining of this section we assume that $\operatorname{Conv}(\Lambda)$ has non-empty interior. The limit set $\Lambda$ is the projection of an acausal closed subset $\widetilde{\Lambda}$ in $\widetilde{\operatorname{Ein}}_{n}$ and $E(\Lambda)$ is the 1-1 projection of a domain $\widetilde{E}(\tilde{\Lambda})$ in $\widetilde{\mathrm{AdS}}_{n+1} \approx \mathbb{R} \times \mathbb{D}^{n}$. Recall that there are two maps $f^{-}, f^{+}$such that $\widetilde{E}(\widetilde{\Lambda})=\left\{(\theta, \mathrm{x}) \mid f^{-}(\mathrm{x})<\theta<f^{+}(\mathrm{x})\right\}$ (cf. Definition 3.3). 
Proposition 3.14. The complement of $\Lambda$ in the boundary $\partial \operatorname{Conv}(\Lambda)$ has two connected components. Both are closed edgeless achronal subsets of $\mathrm{AdS}_{n+1}$. More precisely, in the conformal model their lifting in $\widehat{\mathrm{AdS}}_{n+1}$ are graphs of 1-Lipschitz maps $F^{+}, F^{-}$from $\mathbb{D}^{n}$ into $\mathbb{R}$ such that

$$
f^{-}<F^{-}<F^{+}<f^{+} .
$$

A similar study for $n=2$ but without the assumption that $\Lambda$ is a topological sphere is available in [Bar08b], $\S 8.10$. Before proceeding to the proof of this proposition, we will need to a few lemmas.

Lemma 3.15. Every time-like geodesic of $\operatorname{AdS}_{n+1}$ intersects $\operatorname{Conv}(\Lambda)$.

Proof. Let $D$ be a time-like geodesic in $\operatorname{AdS}_{n+1}$. It is contained in a totally geodesic embedding $A$ of $\mathrm{AdS}_{2}$ in $\mathrm{AdS}_{n+1}$, and the intersection $A \cap \operatorname{Conv}(\Lambda)$ contains the convex hull in $A$ of $\mathrm{Cl}(A) \cap \Lambda$. We are thus reduced to the (easy) case $n=2$. In that case, $A \backslash D$ has two connected components, and each of them contains a connected component of $\partial A$. The boundary $\partial A$ has two connected components $l_{1}$, $l_{2}$, and each of these connected components is an inextendible time-like curve in $\operatorname{Ein}_{1} \subset \operatorname{Ein}_{n+1}$, which therefore intersects $\Lambda$ at an unique point $x_{i}$. Then, the segment $\left[x_{1}, x_{2}\right]$ intersects $D$.

Lemma 3.16. Support hyperplanes in $\mathbb{S}\left(\mathbb{R}^{2, n}\right)$ to $\operatorname{Conv}(\Lambda)$ at points in $\operatorname{AdS}_{n+1}$ are space-like.

Proof. Let $x$ be a point in $\operatorname{AdS}_{n+1} \cap \operatorname{Conv}(\Lambda)$, and let $P$ be a support (projective) hyperplane at $x$ to $\operatorname{Conv}(\Lambda)$. This support hyperplane is a projection $\mathbb{S}\left(v^{\perp}\right)$ for some $v$ in $\mathbb{R}^{2, n}$. If $\mathrm{q}_{2, n}(v)>0$, then $\mathbb{S}\left(v^{\perp}\right)$ disconnects any affine domain, in particular, the affine domain $V$ containing $E(\Lambda) \cup \Lambda$. It follows easily, since $\Lambda$ is a topological sphere, that the affine hyperplane $\mathbb{S}\left(v^{\perp}\right) \cap V$ disconnects $\Lambda$. This is a contradiction since this affine hyperplane is a support hyperplane in $V$ and hence cannot disconnect the convex hull.

If $\mathrm{q}_{2, n}(v)=0$, then the affine hyperplane $V \cap \mathbb{S}\left(v^{\perp}\right)$ is tangent to the hyperboloid $\partial U$ at $\mathbb{S}(v)$ (up to a slight change of affine domain $V$, we can always assume that $\mathbb{S}(v)$ belongs to $V)$. If it disconnects $\Lambda$, we obtain a contradiction as above. If not, it means that $\mathbb{S}(v)$ belongs to $\Lambda$. Write $x$ as a sum $t_{1} x_{1}+\cdots+t_{k} x_{k}$ where $x_{i}$ belongs to $\Lambda: 0=\langle v \mid x\rangle=t_{1}\left\langle v \mid x_{1}\right\rangle+\cdots+t_{k}\left\langle v \mid x_{k}\right\rangle$. Since every $\left\langle v \mid x_{i}\right\rangle$ is a nonpositive number, they all vanish, which implies that $v=x_{i}$ for every $i$. Hence $x=v$; this is a contradiction since $x$ is assumed in $\operatorname{AdS}_{n+1}$.

Proof of Proposition 3.14. Lift $\operatorname{Conv}(\Lambda)$ in $\widetilde{\mathrm{AdS}}_{n+1} \approx \mathbb{R} \times \overline{\mathbb{D}}^{n}$ as a subdomain $\operatorname{Conv}(\tilde{\Lambda})$ in $\widetilde{E}(\widetilde{\Lambda}) \cup \widetilde{\Lambda}$. For every $\mathrm{x}$ in $\mathbb{D}^{n}$, the line $\mathbb{R} \times\{\mathrm{x}\}$ is a time-like geodesic. According to Lemma 3.15 it intersects $\operatorname{Conv}(\tilde{\Lambda})$. Moreover, since this intersection is convex, it contains a geodesic segment $\left[F^{-}(\mathrm{x}), F^{+}(\mathrm{x})\right] \times\{\mathrm{x}\}$. If an element $y$ in 
]$F^{-}(\mathrm{x}), F^{+}(\mathrm{x})[\times\{\mathrm{x}\}$ lies on the boundary of $\operatorname{Conv}(\tilde{\Lambda})$, then every support hyperplane to the convex hull at the projection of this point must contain the projection of the time-like segment $\left[F^{-}(\mathrm{x}), F^{+}(\mathrm{x})\right] \times\{\mathrm{x}\}$ : it contradicts Lemma 3.16. Therefore, the boundary of $\operatorname{Conv}(\widetilde{\Lambda})$ is the union of the graphs of $F^{-}$and $F^{+}$. It follows quite easily that these graphs are closed, hence, $F^{+}$and $F^{-}$are continuous.

Consider the closed subset $\mathscr{E}:=\left\{\mathrm{x} \in \mathbb{D}^{n} \mid F^{-}(\mathrm{x})=F^{+}(\mathrm{x})\right\}$ of $\mathbb{D}^{n}$. For every $\mathrm{x}$ in $\mathbb{D}^{n}$, take a small chart in the Klein model around $\left(F^{+}(\mathrm{x}), \mathrm{x}\right)$ such that $F^{-}$and $F^{+}$expresses locally as graphs of maps from an affine hyperplane into $\mathbb{R}$. Since $\operatorname{Conv}(\Lambda)$ is convex, $F^{+}$is convex and $F^{-}$is concave. It follows that the coincidence locus $\mathcal{E}$ is also open. Since $\mathbb{D}^{n}$ is connected, if $\mathcal{E}$ were not empty we would arrive at the equality $F^{-}=F^{+}$, which is impossible since the interior of $E(\Lambda)$ is not empty. Therefore, according to Lemma 3.12,

$$
f^{-}<F^{-}<F^{+}<f^{+} .
$$

Finally, for every $\mathrm{x}$ in $\mathbb{D}^{n}$, let $\mathbb{S}\left(v^{\perp}\right)$ be a support hyperplane to $\operatorname{Conv}(\Lambda)$ at the projection of $\left(F^{+}(\mathrm{x}), \mathrm{x}\right)$. According to Lemma 3.16, $\mathbb{S}\left(v^{\perp}\right)$ is a totally geodesic embedding of $\mathbb{H}^{n}$. In particular, it lifts as the graph of a 1-Lipschitz map $\varphi_{v}^{+}: \mathbb{D}^{n} \rightarrow$ $\mathbb{R}$. One of the region $\left\{(\theta, \mathrm{y}) \mid \theta>\varphi_{v}^{+}(\mathrm{y})\right\},\left\{(\theta, \mathrm{y}) \mid \theta<\varphi_{v}^{+}(\mathrm{y})\right\}$ is disjoint from $\operatorname{Conv}(\tilde{\Lambda})$, and since $F^{-}(\mathrm{x})<F^{+}(\mathrm{x})=\varphi_{v}^{+}(\mathrm{x})$, it must be the former. Hence on $\mathbb{D}^{n}$ we have $F^{+} \leq \varphi_{p}^{+}$. But since convex domains are intersections of half-spaces containing them, we obtain

$$
F^{+}=\min _{v} \varphi_{v}^{+} .
$$

Since every $\varphi_{v}^{+}$is 1-Lipschitz, the same is true for $F^{+}$. Similarly for $F^{-}$.

We denote the components of $\partial \operatorname{Conv}(\Lambda)$ by $\widetilde{S}^{+}, \widetilde{S}^{-}$.

Remark 3.17. It can be easily inferred from Lemma 3.16 that $\widetilde{S}^{ \pm}$is furthermore acausal, i.e., that $F^{ \pm}$is contracting.

Remark 3.18. Let $v$ be an element $v$ such that $\mathrm{q}_{2, n}(v)>0$. We call AdS-wall and denote by $\partial \mathrm{H}(v)$ the intersection of $\operatorname{AdS}_{n+1}$ with the orthogonal $v^{\perp}$. The half AdS-spaces defined by $v$ are the domains $\mathrm{H}^{+}(v)=\left\{x \in \operatorname{AdS}_{n+1} \mid\langle v \mid x\rangle \geq 0\right\}$ and $\mathrm{H}^{-}(v)=\left\{x \in \operatorname{AdS}_{n+1} \mid\langle v \mid x\rangle \leq 0\right\}=\mathrm{H}^{+}(-v)$. According to Lemma 3.15, the intersection between any AdS-wall $\partial \mathrm{H}(v)$, and $\Lambda_{\rho}$ is a topological $(n-2)$-sphere. Moreover, in a suitable conformal chart $\mathrm{H}^{+}(v)$ is the domain $\left\{\left(\theta, x_{1}, \ldots, x_{n}\right) \in\right.$ $\left.\mathbb{S}^{1} \times \mathbb{D}^{n} \mid x_{n}>0\right\}$. It follows that $\mathscr{S}(v)=\partial \mathrm{H}(v) \cap \operatorname{Conv}\left(\Lambda_{\rho}\right)$ is a topological $(n-1)$-dimensional disk. In particular $\subseteq(v)$ is a compact set and cuts $\operatorname{Conv}\left(\Lambda_{\rho}\right)$ into two parts, $\mathfrak{H}^{ \pm}(v)=\mathrm{H}^{ \pm}(v) \cap \operatorname{Conv}\left(\Lambda_{\rho}\right)$, which we call convex caps. 


\section{Globally hyperbolic AdS spacetimes}

4.1. Cosmological time functions. In any spacetime $(M, g)$, one can define the cosmological time function as follows (see [AGH98]).

Definition 4.1. The cosmological time function of a spacetime $(M, g)$ is the function $\tau: M \rightarrow[0,+\infty]$ defined by

$$
\tau(x):=\sup \left\{L(c) \mid c \in \mathcal{R}^{-}(x)\right\},
$$

where $\mathcal{R}^{-}(x)$ is the set of past-oriented causal curves starting at $x$ and $L(c)$ is the Lorentzian length of the causal curve $c$.

This function behaves in general very badly. For example, in the case of Minkowski space, the cosmological time function is everywhere infinite.

Definition 4.2. A spacetime $(M, g)$ is $C T$-regular with cosmological time function $\tau$ if

(1) $M$ has finite existence time, that is, $\tau(x)<\infty$ for every $x$ in $M$,

(2) for every past-oriented inextendible causal curve $c:[0,+\infty) \rightarrow M$ we have $\lim _{t \rightarrow \infty} \tau(c(t))=0$.

Theorem 4.3 ([AGH98]). CT-regular spacetimes are globally hyperbolic.

A very nice feature of CT-regularity is that it is preserved by isometries (and thus by Galois automorphisms):

Proposition 4.4. Let $(\tilde{M}, \tilde{g})$ be a CT-regular spacetime. Let $\Gamma$ be a torsion-free discrete group of isometries of $(\tilde{M}, \tilde{g})$ preserving the time orientation. Then the action of $\Gamma$ on $(\tilde{M}, \tilde{g})$ is properly discontinuous. Furthermore, the quotient spacetime $(M, g)$ is CT-regular. More precisely, if $\mathrm{p}: \widetilde{M} \rightarrow M$ denote the quotient map, the cosmological times $\tilde{\tau}: \tilde{M} \rightarrow[0,+\infty)$ and $\tau: M \rightarrow[0,+\infty)$ satisfy

$$
\tilde{\tau}=\tau \circ \mathrm{p} .
$$

Sketch of proof. $\Gamma$ clearly preserves the cosmological time and its level sets. These level sets are metric spaces on which $\Gamma$ acts isometrically, and hence, properly discontinuously. It follows quite easily that $\Gamma$ acts properly discontinuously on the entire $\tilde{M}$. Moreover, since $\Gamma$ is discrete and torsion-free, its action on $\tilde{M}$ is free. Therefore, the quotient space is a Lorentzian manifold $M$.

The proof of the identity $\tilde{\tau}=\tau \circ \mathrm{p}$ is straightforward: it follows from the $\Gamma$ invariance of $\tilde{\tau}$ and the fact that inextendible causal curves in $M$ are precisely the projections by p of inextendible causal curves in $\widetilde{M}$. 
4.2. GH-regular AdS spacetimes are CT-regular. Let $\Lambda$ be a non-purely light-like topological achronal $(n-1)$-sphere in $\partial \mathrm{AdS}_{n+1}$.

Proposition 4.5. The GH-regular AdS domain E $(\Lambda)$ is CT-regular.

Proof. Recall that $\Lambda$ is, by definition, the projection of an achronal topological sphere $\tilde{\Lambda} \subset \partial \widetilde{\operatorname{AdS}}_{n+1}$, and that $E(\Lambda)$ is the projection of the invisible domain $\widetilde{E}(\widetilde{\Lambda})$ of $\tilde{\Lambda}$ in $\widetilde{\operatorname{AdS}}_{n+1} \cup \partial \widetilde{\mathrm{AdS}}_{n+1}$. We will prove that $\widetilde{E}(\widetilde{\Lambda})$ has regular cosmological time. Since the projection of $\widetilde{E}(\widetilde{\Lambda})$ on $E(\Lambda)$ is one-to-one (Lemma 3.4), this will imply that $E(\Lambda)$ also has regular cosmological time. We denote by $\tilde{\tau}$ the cosmological time of $\widetilde{E}(\widetilde{\Lambda})$.

Let $x$ be a point in $\widetilde{E}(\tilde{\Lambda})$. On the one hand, according to Corollary 3.7, $\mathrm{Cl}(\widetilde{E}(\tilde{\Lambda}))$ is a compact subset of $\widetilde{\operatorname{AdS}}_{n+1} \cup \partial \widetilde{\operatorname{AdS}}_{n+1}$, and the intersection $\mathrm{Cl}(\widetilde{E}(\widetilde{\Lambda})) \cap \partial \widetilde{\operatorname{AdS}}_{n+1}$ equals $\widetilde{\Lambda}$. On the other hand, since $x$ is in the invisible domain of $\widetilde{\Lambda}$, the set $J^{-}(x)$ is disjoint from $\tilde{\Lambda}$. Therefore $J^{-}(x) \cap \operatorname{Cl}(\widetilde{E}(\widetilde{\Lambda}))$ is a compact subset of $\widetilde{\operatorname{AdS}_{n+1}}$. Therefore $J^{-}(x) \cap \operatorname{Cl}(\widetilde{E}(\widetilde{\Lambda}))$ is conformally equivalent to a compact causally convex domain in $\left(\mathbb{R} \times \mathbb{D}^{n},-d \theta^{2}+d s^{2}\right)$, with a bounded conformal factor since everything is compact. It follows that the length of the past-directed causal curves starting at $x$ contained in $\widetilde{E}(\widetilde{\Lambda})$ is bounded (in other words, $\tilde{\tau}(x)$ is finite) and that, for every past-oriented inextendible causal curve $c:[0,+\infty) \rightarrow \widetilde{E}(\tilde{\Lambda})$ with $c(0)=x$, one has $\tilde{\tau}(c(t)) \rightarrow 0$ when $t \rightarrow \infty$. This shows that $\widetilde{E}(\widetilde{\Lambda})$ has regular cosmological time.

Hence, according to Theorem 4.3, GH-regular domains are globally hyperbolic.

4.3. GH-regular and quasi-Fuchsian representations. Let $\Lambda$ be a non-purely light-like topological achronal $(n-1)$-sphere in $\partial \mathrm{AdS}_{n+1}$, and $\Gamma$ a torsion-free discrete subgroup of $\mathrm{SO}_{0}(2, n)$ preserving $\Lambda$. According to Proposition 4.4, the quotient space $\Gamma \backslash E(\Lambda)$ is globally hyperbolic.

Conversely, in his celebrated 1990 preprint [Mes07] (see also [ABB ${ }^{+}$07]), G. Mess ${ }^{1}$ proved that any globally hyperbolic spatially compact AdS spacetime embeds isometrically in such a quotient space $\Gamma \backslash E(\Lambda)$.

Definition 4.6. Let $\Gamma$ be a torsion-free discrete group. A representation $\rho: \Gamma \rightarrow$ $\mathrm{SO}_{0}(2, n)$ is called GH-regular if it is faithful, discrete and preserves a GH-regular domain $E(\Lambda)$ in $\partial \mathrm{AdS}_{n+1}$. If moreover the $(n-1)$-sphere $\Lambda$ is acausal, then the representation is strictly GH-regular.

Definition 4.7. A (strictly) GH-regular representation $\rho: \Gamma \rightarrow \mathrm{SO}_{0}(2, n)$ is (strictly) $G H C$-regular if the quotient space $\rho(\Gamma) \backslash E(\Lambda)$ is spatially compact.

Hence a reformulation of Mess result is:

\footnotetext{
${ }^{1}$ Mess only deals with the case where $n=2$, but his arguments also apply in higher dimension. For a detailed proof see [Bar08b], Corollary 11.2.
} 
Proposition 4.8. A representation $\rho: \Gamma \rightarrow \mathrm{SO}_{0}(2, n)$ is GHC-regular if and only if it is the holonomy of a GHC AdS spacetime.

Let $M=\rho(\Gamma) \backslash E(\Lambda)$ be a GHC AdS spacetime. In $\S 3.3$ we proved that $E(\Lambda)$ contains a convex subdomain $\operatorname{Conv}(\Lambda) \backslash \Lambda$, whose boundary is the union of two connected components $\widetilde{S}^{ \pm}$. Denote by $S^{+}, S^{-}$their projections in $M=\rho(\Gamma) \backslash E(\Lambda)$.

Lemma 4.9. $S^{ \pm}$are Cauchy hypersurfaces in $M$.

Proof. Since $\widetilde{S}^{ \pm}$is homeomorphic to $\mathbb{R}^{n}$ the quotient $S^{ \pm}=\widetilde{S}^{ \pm}$is a $K(\Gamma, 1)$ space. But any Cauchy surface in $M$ is also a $K(\Gamma, 1)$ space, and since $M$ is GHC, these Cauchy surfaces are compact. Consequently, every $K(\Gamma, 1)$ space is compact, and the lemma follows.

As a corollary, the projection of $\operatorname{Conv}(\Lambda) \backslash \Lambda$ in $M$ is the compact domain bounded by the two disjoint Cauchy hypersurfaces $S^{ \pm}$. We denote this set by $C(M)$ and call it the convex core of $M$.

In this paper, we will focus on a special case of strictly GHC-regular representations: the case of quasi-Fuchsian representations.

Definition 4.10. A strictly GHC-regular representation $\rho: \Gamma \rightarrow \mathrm{SO}_{0}(2, n)$ is quasiFuchsian if $\Gamma$ is isomorphic to a uniform lattice in $\mathrm{SO}_{0}(1, n)$.

This terminology is motivated by the analogy with the hyperbolic case.

There is a particular case: the case where $\Lambda$ is a "round sphere" in $\partial \operatorname{AdS}_{n+1}$, i.e., the boundary of a totally geodesic space-like hypersurface $\mathbb{S}\left(v^{\perp}\right) \cap \operatorname{AdS}_{n+1}$ :

Definition 4.11. A Fuchsian representation $\rho: \Gamma \rightarrow \mathrm{SO}_{0}(2, n)$ is the composition of the natural inclusions $\Gamma \subset \mathrm{SO}_{0}(1, n)$ and $\mathrm{SO}_{0}(1, n) \subset \mathrm{SO}_{0}(2, n)$, where in the latter $\mathrm{SO}_{0}(1, n)$ is considered as the stabilizer in $\mathrm{SO}_{0}(2, n)$ of a point in $\mathrm{AdS}_{n+1}$.

In other words, a quasi-Fuchsian representation is Fuchsian if and only if it admits a global fixed point in $\operatorname{AdS}_{n+1}$.

\section{Anosov representations are strictly GHC-regular}

\subsection{Anosov representations}

5.1.1. General definition. Let $N$ be a manifold equipped with a non-singular flow $\Phi^{t}$ and an auxiliary Riemannian metric $\|\cdot\|$.

Definition 5.1. A closed subset $F \subseteq N$ is $\Phi^{t}$-hyperbolic if it is $\Phi^{t}$-invariant and the tangent bundle of $N$ admits a decomposition $T N=\Delta \oplus E^{s s} \oplus E^{u u}$ over $F$ such that, for some positive constants $a, b$, 
- the line bundle $\Delta$ is tangent to the flow,

- for any vector $v$ in $E^{s s}$ over a point $p$ of $N$ and for any positive $t$,

$$
\left\|d_{p} \Phi^{t}(v)\right\| \leq b e^{-a t}\|v\|
$$

- for any vector $v$ in $E^{u u}$ over a point $p$ of $N$ and for any negative $t$,

$$
\left\|d_{p} \Phi^{t}(v)\right\| \leq b e^{a t}\|v\| .
$$

If $F$ is the entire manifold $N$, the flow $\Phi^{t}$ is Anosov.

The typical example of an Anosov flow, and the only one that we will consider here, is the geodesic flow on the unit tangent bundle to a negatively curved Riemannian manifolds.

Let $\Gamma$ be the fundamental group of $N$. Let $Y$ be a manifold, and $G$ be a Lie group acting smoothly on $Y$. Given any representation $\rho: \Gamma \rightarrow G$ one can construct the associated flat bundle $E_{\rho}$ over $N$ as the quotient of the product $\tilde{N} \times Y$ by the natural action of $\Gamma$. The projection function from $E_{\rho}$ to $N$ is denoted by $\pi_{\rho}$. The bundle $E_{\rho}$ inherits a flow $\Phi_{\rho}^{t}$ from the lifting $\widetilde{\Phi}^{t}$ of $\Phi^{t}$ on $\tilde{N}$.

Definition 5.2. A representation $\rho: \Gamma \rightarrow G$ is $(G, Y)$-Anosov if the flat bundle $\pi_{\rho}: E_{\rho} \rightarrow N$ admits a continuous section $s: N \rightarrow E_{\rho}$ such that the image of $s$ is an invariant hyperbolic subset for $\Phi_{\rho}^{t}$.

A very nice feature of Anosov representations is the following proposition, which is consequence of the stability property of closed hyperbolic set (see [Lab06], Proposition 2.1 for a proof):

Theorem 5.3. Let $N$ a compact manifold endowed with an Anosov flow $\Phi^{t}$. The set of $(G, Y)$-Anosov representations from $\Gamma=\pi_{1}(N)$ to $G$ is open in the space of representations of $\Gamma$ in $G$, usually denoted by $\operatorname{Rep}(\Gamma, G)$ (endowed with the compactopen topology).

5.1.2. Anosov AdS representations. Here we are concerned with the case $(G, Y)=$ $\left(\mathrm{SO}_{0}(2, n), y\right)$ where $y$ is the open subset of $\operatorname{Ein}_{n} \times \operatorname{Ein}_{n}$ made of the pairs of points that can be joined by a space-like geodesic. Given a $\left(\mathrm{SO}_{0}(2, n), y\right)$-Anosov representation, the section $s: N \rightarrow E_{\rho}$ defining the Anosov property lifts to a map $\ell_{\rho}: \tilde{N} \rightarrow y$ which is $\Gamma$-equivariant, i.e., $\rho_{g} \circ \ell_{\rho}=\ell_{\rho} \circ g$, and $\Phi^{t}$-invariant. This mapping can be decomposed in $\ell_{\rho}=\left(\ell_{\rho}^{+}, \ell_{\rho}^{-}\right)$where $\ell_{\rho}^{+}$(resp. $\left.\ell_{\rho}^{-}\right)$are two mappings from $\tilde{N}$ to $\operatorname{Ein}_{n}$.

Remark 5.4. An equivalent way to formulate the $(G, y)$-Anosov property is to require the existence of continuous maps $\ell_{\rho}^{ \pm}: \widetilde{N} \rightarrow \operatorname{Ein}_{n}$ and of a family of Riemannian metrics $g^{p}$ depending continuously on $p \in \tilde{N}$ and defined in a neighborhood of $\ell_{\rho}^{ \pm}(p)$ in $\operatorname{Ein}_{n}$ such that 
(1) this family is $\Gamma$-equivariant, i.e., for every $\gamma$ in $\Gamma$,

$$
g^{\gamma p}(d \rho(\gamma) w, d \rho(\gamma) w)=g^{p}(w, w),
$$

where $w$ belongs to $\mathrm{T}_{\ell^{ \pm}(p)} \operatorname{Ein}_{n}$ and $d \rho(\gamma)$ is the differential of $\rho(\gamma)$ at $\ell^{ \pm}(p)$;

(2) the family increases (resp. decreases) exponentially along positive (resp. negative) orbits of $\widetilde{\Phi}^{t}$, i.e., for some $a, b>0$, if $w$ is a vector tangent at $\ell_{\rho}^{+}(p)$ (resp. $\left.\ell_{\rho}^{-}(p)\right)$ to $\operatorname{Ein}_{n}$, then

$$
\begin{aligned}
& g^{\widetilde{\Phi}^{t}(p)}(w, w) \geq b^{-1} \exp (a t) g^{p}(w, w), \\
& g^{\widetilde{\Phi}^{t}(p)}(w, w) \leq b \exp (-a t) g^{p}(w, w) .
\end{aligned}
$$

5.1.3. A criterion for a representation to be Anosov. A technical difficulty arising when one wants to prove that a representation is Anosov is to ensure the exponential decay. The following proposition shows that this property follows from the compactness of the ambient manifold $N$ of the Anosov flow and a weaker contraction property somewhere along the orbit.

Proposition 5.5. Let $\rho: \Gamma \rightarrow \mathrm{SO}_{0}(2, n)$ be a representation, and assume the existence of continuous maps $\ell_{\rho}^{ \pm}: \tilde{N} \rightarrow \operatorname{Ein}_{n}$ and of a continuous and $\Gamma$-equivariant family of Riemannian metrics $g^{p}$ defined in a neighborhood of $\ell_{\rho}^{ \pm}(p)$ in $\operatorname{Ein}_{n}$. Suppose moreover that the following assumption is satisfied:

$\left(2^{\prime}\right)$ For all points $p$ in $\tilde{N}$, there exists a time $t>0$ such that

$$
\begin{aligned}
& g^{\widetilde{\Phi}^{t}(p)}(w, w) \geq 2 g^{p}(w, w) \quad \text { for all } w \in \mathrm{T}_{\ell_{\rho}^{+}(p)} \operatorname{Ein}_{n}, \\
& g^{\widetilde{\Phi}^{t}(p)}(w, w) \leq 2 g^{p}(w, w) \quad \text { for all } w \in \mathrm{T}_{\ell_{\rho}^{-}(p)} \operatorname{Ein}_{n} .
\end{aligned}
$$

Then $\rho$ is $\left(\mathrm{SO}_{0}(2, n), y\right)$-Anosov.

Proof. We simply prove that the hypotheses above imply the exponential decay and exponential expansion expressed in Remark 5.4. Let $\pi_{\rho}: E_{\rho} \rightarrow N$ be the flat $\operatorname{Ein}_{n^{-}}$ bundle associated to $\rho$, and let $s^{ \pm}: N \rightarrow E_{\rho}$ be the sections induced by $\ell_{\rho}^{ \pm}$. Since the family $\left(g^{p}\right)_{(p \in \tilde{N})}$ is $\Gamma$-equivariant, it induces for every $p$ in $N$ a metric $g_{ \pm}^{p}$ on the fiber $\pi^{-1}(p)$ near $s^{-}(p)$ and $s^{+}(p)$. Denote by $V^{ \pm}(p)$ the vertical tangent bundle at $s^{ \pm}(p)$. For every $p$ in $N$ and every $t$ define

$$
\alpha^{-}(p, t)=\sup _{w \in V^{-}(p)} \frac{g_{-}^{\Phi^{t}(p)}(w, w)}{g \underline{p}(w, w)}, \quad \alpha^{+}(p, t)=\inf _{w \in V^{-}(p)} \frac{g_{+}^{\Phi^{t}(p)}(w, w)}{g_{+}^{p}(w, w)} .
$$

Obviously, for $s, t>0$,

$$
\begin{aligned}
& \alpha^{-}(p, t+s) \leq \alpha^{-}(p, s) \alpha^{-}\left(\Phi^{s}(p), t\right), \\
& \alpha^{+}(p, t+s) \geq \alpha^{+}(p, s) \alpha^{+}\left(\Phi^{s}(p), t\right) .
\end{aligned}
$$


By hypothesis, and since $N$ is compact, there is a finite covering $\left(U_{i}\right)_{(1 \leq i \leq k)}$ of $N$, and a sequence $\left(T_{i}\right)_{(1 \leq i \leq k)}$ such that for any $i$ in $\{1, \ldots, k\}$ and any $p$ in $U_{i}$ we have $\alpha^{-}\left(p, T_{i}\right) \leq 1 / 2$. Put $T=\sup \left\{T_{i} \mid 1 \leq i \leq k\right\}$ and $a=\sup \left\{\alpha^{-}(p, t) \mid t \in\right.$ $[0, T], p \in N\}$. For any $p$ in $N$ there exist sequences $\left(t_{j}\right)_{(0 \leq j \leq J)}$ and $\left(i_{j}\right)_{(0 \leq j \leq J)}$ such that $t_{0}=0, t_{J-1} \leq t \leq t_{J}, \Phi^{t_{j}}(p)$ lies in $U_{i_{j}}$ and $t_{j+1}=t_{j}+T_{i_{j}}$. Then

$$
\begin{aligned}
\alpha^{-}(p, t) & \leq \alpha^{-}\left(p, T_{i_{0}}\right) \alpha^{-}\left(\Phi^{t_{1}}(p), T_{i_{1}}\right) \ldots \alpha^{-}\left(\Phi^{t_{J-1}}(p), t-t_{J-1}\right) \\
& \leq(1 / 2)^{J-1} a \\
& \leq a(1 / 2)^{t / T-1}
\end{aligned}
$$

since $t \leq J T$. It follows that $\alpha^{-}(p, t)$ decreases exponentially with $t$. Similarly, $\alpha^{+}(p, t)$ increases exponentially with $t$. The proposition follows.

5.1.4. Basic properties of the geodesic flow. As we mentioned previously, we only consider the case where the Anosov flow is the geodesic flow on the unit tangent bundle $N=\mathrm{T}^{1} \Sigma$ over a negatively curved Riemannian manifold $\Sigma$. Let $\widetilde{\Sigma}$ denote the universal covering of $\Sigma$, and let $\partial \widetilde{\Sigma}$ be its boundary as a hyperbolic space.

The manifold $N$ is the quotient of the unit tangent bundle $\mathrm{T}^{1} \widetilde{\Sigma}$ by the fundamental group $\Gamma$ of $\Sigma$ (except in the case $n=2$, the discussion of which is left to the reader). The geodesic flow $\Phi^{t}$ is the projection of the geodesic flow $\widetilde{\Phi}^{t}$ of $\mathrm{T}^{1} \widetilde{\Sigma}^{n}$. Let us recall a few well-known properties of geodesic flows on negatively curved manifolds and fix some notation:

(1) The orbit of a tangent vector $(x, v)$ in $\mathrm{T}^{1} \widetilde{\Sigma}$ by $\widetilde{\Phi}^{t}$ is the set of points $\left(x^{t}, v^{t}\right)$, where $x^{t}$ is the unit speed geodesic starting at $x$ with speed $v$, and $v^{t}$ is the unit tangent to this geodesic at $x^{t}$,

(2) We denote by $\ell^{+}(x, v)$ the future extremity of the geodesic tangent to $(x, v)$ in the boundary $\partial \widetilde{\Sigma}$, and by $\ell^{-}(x, v)$ the past extremity of this geodesic. The fibers of the maps $\ell^{+}$(resp. $\ell^{-}$) are called the stable leaves (resp. unstable leaves).

(3) If two unit vectors $(x, v)$ and $\left(x^{\prime}, v^{\prime}\right)$ belong to the same stable leaf, then there exists a time delay $T$ such that the distance between $\widetilde{\Phi}^{t+T}(x, v)$ and $\widetilde{\Phi}^{t}\left(x^{\prime}, v^{\prime}\right)$ tends to 0 exponentially fast when $t \rightarrow+\infty$.

(4) A (unit speed) geodesic in $\widetilde{\Sigma}$ is characterized by its two distinct extremities. Hence, the map $\left(\ell^{+}, \ell^{-}\right): \mathrm{T}^{1} \widetilde{\Sigma} \rightarrow \partial \widetilde{\Sigma} \times \partial \widetilde{\Sigma}$ induces an identification between the orbit space of $\widetilde{\Phi}$ and $\partial \widetilde{\Sigma} \times \partial \widetilde{\Sigma} \backslash \mathfrak{D}$ where $\mathfrak{D}$ is the diagonal.

(5) Every element $\gamma$ of $\Gamma$ is loxodromic: it admits one attractive fixed point $x_{\gamma}^{+}$ in $\partial \widetilde{\Sigma}$ and one repelling fixed point $x_{\gamma}^{-}$. The geodesic with extremities $x_{\gamma}^{+}$ and $x_{\gamma}^{-}$is then the unique geodesic of $\widetilde{\Sigma}$ preserved by $\gamma$. Moreover, there exists a real number $T>0$ such that for every vector $(x, v)$ tangent to the $\gamma$-invariant geodesic, and such that $\ell^{+}(x, v)=x_{\gamma}^{+}, \ell^{-}(x, v)=x_{\gamma}^{-}$we have $\widetilde{\Phi}^{T}(x, v)=\gamma(x, v)$.

(6) The set of attractive fixed points of elements of $\Gamma$ is dense in $\partial \widetilde{\Sigma}$. 
(7) Periodic geodesics are dense in $N$, i.e., pairs $\left(x_{\gamma}^{+}, x_{\gamma}^{-}\right)$are dense in $\partial \widetilde{\Sigma} \times \partial \widetilde{\Sigma} \backslash \mathfrak{D}$.

(8) $\Gamma$ acting on $\partial \widetilde{\Sigma}$ is a convergence group: from any sequence $\left(\gamma_{n}\right)_{(n \in \mathbb{N})}$ in $\Gamma$ we can extract a subsequence $\left(\gamma_{n_{k}}\right)_{(k \in \mathbb{N})}$ for which there are two points $x^{+}, x^{-}$in $\partial \widetilde{\Sigma}$ such that

- $\left(\gamma_{n_{k}}\right)_{(k \in \mathbb{N})}$ converges uniformly on compact subsets of $\partial \widetilde{\Sigma} \backslash\left\{x^{-}\right\}$to the constant map taking the value $x^{+}$;

- $\left(\gamma_{n_{k}}^{-1}\right)_{(k \in \mathbb{N})}$ converges uniformly on compact subsets of $\partial \widetilde{\Sigma} \backslash\left\{x^{+}\right\}$to the constant map taking the value $x^{-}$.

5.2. Fuchsian representations are Anosov. In this $\S$ we prove that Fuchsian representations, i.e., the representations that admit a fixed point in the anti-de Sitter space (cf. Definition 4.11) are $\left(\mathrm{SO}_{0}(2, n), y\right)$-Anosov. It should be noted that this statement is actually a special case of Proposition 3.1 in [Lab06], but the proof we give has the advantage to introduce notations and arguments that will be useful later. In this setting, $\widetilde{\Sigma}$ denotes the hyperbolic space $\mathbb{H}^{n}$, and the group $\Gamma$ is a lattice in $\operatorname{SO}_{0}(1, n)$.

5.2.1. De Sitter domains in $\operatorname{Ein}_{n}$. For any point $x$ in the anti-de Sitter space $\operatorname{AdS}_{n+1}$, the associated de Sitter domain $\partial U(x)$ (cf. Remark 2.10) is the open subset of the Einstein space $\operatorname{Ein}_{n}$ containing the limits of space-like geodesics starting at $x$. A unit space-like vector $v$ tangent to $x$ satisfies $\langle x \mid v\rangle=0$ and $\mathrm{q}_{2, n}(v)=1$. Hence, the vector $x+v$ belongs to the isotropic cone $\ell_{n}$, and moreover it corresponds to the limit $\ell^{+}(x, v)$ in the Einstein space (see $\left.\S 2.8\right)$. Hence the de Sitter domain $\partial U(x)$ is nothing but the projection on the sphere $\mathbb{S}\left(\mathbb{R}^{2, n}\right)$ of the set

$$
U_{x}:=\left\{x+v \mid v \in\{x\}^{\perp}, \mathrm{q}_{2, n}(v)=1\right\} \subseteq \mathcal{C} .
$$

An inverse map for this projection can be constructed from the mapping $s_{x}: \mathbb{R}^{2, n} \backslash$ $\{x\}^{\perp} \rightarrow \mathbb{R}^{2, n}$ which maps a point $y \in \mathbb{R}^{2, n} \backslash\{x\}^{\perp}$ to the unique collinear point $s_{x}(y)$ in $H_{x}=\{z \mid\langle z \mid x\rangle=-1\}$, i.e., $s_{x}(y)=-y /\langle y \mid x\rangle$. This map induces a diffeomorphism $\tilde{s}_{x}: \partial U(x) \subseteq \operatorname{Ein}_{n} \rightarrow U_{x}$.

5.2.2. Construction of the metric. For every choice of a point $V$ in $\operatorname{AdS}_{n+1}$ such that $\langle x \mid V\rangle=0$, we can define a unit time-like vector field $\tau^{x, V}$ on $\mathcal{U}_{x}$ as follows. For any $\zeta \in U_{x}$, we let $v=\zeta-x$ be the corresponding tangent vector in $\mathrm{T}_{x} \mathrm{AdS}_{n+1}$ and set

$$
\tau_{\zeta}^{x, V}:=\frac{V-\langle V \mid v\rangle v}{\langle V \mid v\rangle^{2}+1}
$$

We construct a metric $\bar{g}_{\zeta}^{x, V}$ on $\mathrm{T}_{\zeta} U_{x}$ by reversing the sign of $\tau_{\zeta}^{x, V}$ in the metric induced by $\mathrm{q}_{2, n}$ : for every tangent vector $w$ in $\mathrm{T}_{\zeta} U_{x}$, we let

$$
\bar{g}_{\zeta}^{x, V}(w, w):=\mathrm{q}_{2, n}(w, w)+2\left\langle w \mid \tau_{\zeta}^{x, V}\right\rangle^{2}
$$


Finally, let us denote by $g^{x, V}$ the Riemannian metric on $\partial U(x)$ obtained by pullingback $\bar{g}^{x, V}$ by the section $\tilde{s}_{x}$ introduced earlier.

Remarks 5.6. (1) In the terminology of [BB09] the metric $g^{x, V}$ is the Wick rotation performed on the de Sitter metric of $\partial U(x)$ along the gradient of the time function $\zeta \rightarrow\langle V \mid \zeta\rangle$.

(2) The previous construction is $\mathrm{SO}_{0}(2, n)$-equivariant in the sense that

$$
\gamma \partial U(x)=\partial U(\gamma(x)) \quad \text { and } \quad g_{\gamma(\zeta)}^{\gamma x, \gamma V}(\mathrm{~d} \gamma(w), \mathrm{d} \gamma(w))=g_{\zeta}^{x, V}(w, w)
$$

for any isometry $\gamma$ of $\mathbb{R}^{2, n}$.

5.2.3. The inclusion is Anosov. The group $\rho_{0}(\Gamma)$ preserves an element $V$ of $\operatorname{Ad} S_{n+1}$ and the stable space-like hypersurface $\mathbb{S}\left(V^{\perp}\right) \cap \mathrm{AdS}_{n+1}$, which is isometric to the hyperbolic space $\mathbb{H}^{n}$. This gives a natural inclusion $\mathrm{T}^{1} \mathbb{H}^{n} \subseteq \mathcal{E}^{1} \mathrm{Ad} \mathrm{S}_{n+1}$. We define the maps $\ell_{\rho_{0}}^{ \pm}$from the unit tangent bundle $\mathrm{T}^{1} \mathbb{H}^{n}$ to $\operatorname{Ein}_{n}$ by restriction of $\ell^{ \pm}$. These maps are both $\widetilde{\Phi}^{t}$-invariant and $\Gamma$-equivariant. Since $\mathbb{H}^{n} \subseteq \operatorname{AdS}_{n+1}$ is space-like, $\ell_{\rho_{0}}^{+}(x, v)$ and $\ell_{\rho_{0}}^{-}(x, v)$ are joined by a space-like geodesic, implying that the map $\ell_{\rho_{0}}:=\left(\ell_{\rho_{0}}^{-}, \ell_{\rho_{0}}^{+}\right)$takes its value in $y \subseteq \operatorname{Ein}_{n} \times \operatorname{Ein}_{n}$.

Thus, in order to prove that the representation $\rho_{0}$ is Anosov, we only need to check the hyperbolicity property, as formulated in Remark 5.4, for the family of metrics $g^{(x, v)}:=g^{x, V}$. This is the goal of the following proposition. We only prove the expanding property at $\ell_{\rho_{0}}^{+}(x, v)$, the contracting property at $\ell_{\rho_{0}}^{-}(x, v)$ being similar.

Proposition 5.7. Let $v$ be a unit tangent vector to a point $x$ in $\mathbb{H}^{n}$, and let $v$ be tangent to $\operatorname{Ein}_{n}$ at $\ell_{\rho_{0}}^{+}(x, v)$. Then $g^{\widetilde{\Phi}_{\rho_{0}}^{t}(x, v)}(v, v)=\exp (2 t) g^{(x, v)}(v, v)$.

Proof. Let $(x, v)$ be an element of $\mathrm{T}^{1} \mathbb{H}^{n}$, and $x^{t}$ be the base-point of $\widetilde{\Phi}^{t}(x, v)$, i.e., $x^{t}=(\cosh t) x+(\sinh t) v$. While the limit vector $\zeta=\ell_{\rho_{0}}^{+}\left(\widetilde{\Phi}^{t}(x, v)\right)$ does not change in the Einstein universe, its representative element in $u_{x^{t}} \subset \varphi_{n} \subset \mathbb{R}^{2, n}$ varies with $t$. The exponential expanding behavior comes from the changes in the derivative of the maps $s_{x^{t}}$.

The representative element of $\zeta=\ell_{\rho_{0}}^{+}(x, v)$ in $U_{x}$ is $x+v$. The tangent vector $v$ is the image under the derivative of $s_{x}$ of a vector $w$ tangent to $U_{x} \cap \ell_{n}$. In particular, we have $\langle w \mid x\rangle=\langle w \mid v\rangle=0$. Its representative element in $\boldsymbol{U}_{x^{t}}$ is the image $w^{t}$ of $w$ by the derivative of $s_{x}$ at $x+v$, i.e.,

$$
w^{t}=\mathrm{d}_{x+v} s_{x^{t}}(w)=\frac{(x+v)\left\langle w \mid x^{t}\right\rangle-w\left\langle x+v \mid x^{t}\right\rangle}{\left\langle x+v \mid x^{t}\right\rangle^{2}}=\frac{0+w e^{-t}}{e^{-2 t}}=e^{t} w .
$$

Since $\langle V \mid v\rangle=0$, the vector $\tau_{\zeta}^{x^{t}, V}$ is simply $V$, and does not depend on the time $t$. The proposition follows by plugging the above equation in the definition of the metric. 
5.3. Anosov representations are strictly GHC-regular. We now proceed to the proof that Anosov representations are strictly GHC-regular, i.e., the first inclusion in Theorem 1.2. In the following, we assume that $\rho: \Gamma \rightarrow \mathrm{SO}_{0}(2, n)$ is a $\left(\mathrm{SO}_{0}(2, n), y\right)$ Anosov representation.

Lemma 5.8. The mapping $\ell_{\rho}^{+}\left(\right.$resp. $\left.\ell_{\rho}^{-}\right)$is constant along the leaves of the stable (resp. unstable) foliation of $\tilde{N}$.

Proof. Follows from 5.1.4(3) and from the compactness of $N=\Gamma \backslash \mathrm{T}^{1} \widetilde{\Sigma}$.

Therefore, the maps $\ell_{\rho}^{ \pm}$induce $\Gamma$-equivariant maps $\bar{\ell}_{\rho}^{ \pm}: \partial \widetilde{\Sigma} \rightarrow \operatorname{Ein}_{n}$.

Proposition 5.9. Let $\alpha$ be the map from $\mathrm{T}^{1} \widetilde{\Sigma}$ to itself which sends the vector $(x, v)$ to $(x,-v)$. Then $\ell_{\rho}^{+}=\ell_{\rho}^{-} \circ \alpha$.

In order to prove this proposition we need a few lemmas.

Lemma 5.10. Let $\gamma$ be an element of $\Gamma$. Then $\bar{\ell}_{\rho}^{+}\left(x_{\gamma}^{+}\right)\left(\right.$resp. $\left.\bar{\ell}_{\rho}^{-}\left(x_{\gamma}^{-}\right)\right)$is an attractive (resp. repelling) fixed point of $\rho(\gamma)$.

Proof. This is a general fact for Anosov representations. See for example [Lab06], Proposition 3.2.

Lemma 5.11. For every $\gamma$ in $\Gamma$, the image $\rho(\gamma)$ admits exactly two attractive fixed points in $\operatorname{Ein}_{n}$ opposite to each other.

Proof. Let $x^{+}$be an attractive fixed point of $\rho(\gamma)$ in $\operatorname{Ein}_{n}$ : there exists a neighborhood $U$ of $x^{+}$in $\operatorname{Ein}_{n}$ such that for all $y \in U, \rho(\gamma)^{n} y \rightarrow x^{+}$. The convex hull of $U$ in $\mathbb{S}\left(\mathbb{R}^{2, n}\right)$ satisfies the same property, but it is also a neighborhood of $x^{+}$in $\mathbb{S}\left(\mathbb{R}^{2, n}\right)$. This follows from the fact that in any affine chart of $\mathbb{S}\left(\mathbb{R}^{2, n}\right)$ around $x$, the Einstein space is a one-sheeted hyperboloid. Hence $x^{+}$is also an attractive fixed point in the projective space $\mathbb{S}\left(\mathbb{R}^{2, n}\right)$. The lemma follows since attractive fixed points of projective automorphisms of $\mathbb{P}\left(\mathbb{R}^{2, n}\right)$ are unique.

Proof of Proposition 5.9. It follows from Lemmas 5.10, 5.11 that $\ell_{\rho}^{+}(x, v)= \pm \ell_{\rho}^{-}$。 $\alpha(x, v)$ when the geodesic tangent to $(x, v)$ is preserved by a non-trivial element of $\Gamma$. Since periodic orbits are dense (item (7) in $\S 5.1 .4$ ) this equality holds everywhere. By continuity of $\ell^{ \pm}$we have either the equality $\ell_{\rho}^{+}(x, v)=\ell_{\rho}^{-} \circ \alpha(x, v)$ everywhere, or $\ell_{\rho}^{+}(x, v)=-\ell_{\rho}^{-} \circ \alpha(x, v)$ everywhere.

Assume that the latter case holds. Since $\mathrm{T}^{1} \widetilde{\Sigma}$ is simply connected, we can lift $\ell_{\rho}^{ \pm}$ to maps $\tilde{\ell}_{\rho}^{ \pm}: \mathrm{T}^{1} \widetilde{\Sigma} \rightarrow \widetilde{\operatorname{Ein}}_{n+1} \approx \mathbb{R} \times \mathbb{S}^{n-1}$. We write $\ell_{\rho}^{ \pm}=\left(\theta_{\rho}^{ \pm}, \mathrm{x}_{\rho}^{ \pm}\right)$. Then the equality $\ell_{\rho}^{+}(x, v)=-\ell_{\rho}^{-} \circ \alpha(x, v)$ means that we can select the liftings $\tilde{\ell}_{\rho}^{ \pm}$so that for every $(x, v)$ in $\mathrm{T}^{1} \widetilde{\Sigma}$ we have $\mathrm{x}_{\rho}^{-}(x, v)=-\mathrm{x}_{\rho}^{+}(x, v)$ and $\theta_{\rho}^{-}(x, v)=\theta_{\rho}^{+}(x, v)-\pi$. 
Fix an element $\left(x_{0}, v_{0}\right)$ in $\mathrm{T}^{1} \widetilde{\Sigma}$. Denote $l^{-}\left(x_{0}, v_{0}\right)$ by $p_{0}$. For every $p$ in $\partial \widetilde{\Sigma} \backslash\left\{p_{0}\right\}$ there is an element $(x, v)$ of $\mathrm{T}^{1} \widetilde{\Sigma}$ such that $\ell^{-}(x, v)=p$ and $\ell^{+}(x, v)=\ell^{+}\left(x_{0}, v_{0}\right)$. Then, since $\ell_{\rho}(x, v)=\left(\ell_{\rho}^{+}(x, v), \ell_{\rho}^{-}(x, v)\right)$ takes value in $\mathcal{Y}$, there is a spacelike geodesic in $\widetilde{\operatorname{AdS}}_{n+1}$ joining $\tilde{\ell}_{\rho}^{-}(p)=\left(\theta_{\rho}^{-}(x, v), \mathrm{x}_{\rho}^{-}(x, v)\right)$ to $\tilde{\ell}_{\rho}^{+}\left(x_{0}, v_{0}\right)=$ $\left(\theta_{\rho}^{+}\left(x_{0}, v_{0}\right), \mathrm{x}_{\rho}^{+}\left(x_{0}, v_{0}\right)\right)$ In other words, we must have $d\left(\mathrm{x}_{\rho}^{-}(x, v), \mathrm{x}_{\rho}^{+}\left(x_{0}, v_{0}\right)\right)>$ $\left|\theta_{\rho}^{-}(x, v)-\theta_{\rho}^{+}\left(x_{0}, v_{0}\right)\right|$. Since we have $\mathrm{x}_{\rho}^{-}\left(x_{0}, v_{0}\right)=-\mathrm{x}_{\rho}^{+}\left(x_{0}, v_{0}\right)$ and $\theta_{\rho}^{-}\left(x_{0}, v_{0}\right)=$ $\theta_{\rho}^{+}\left(x_{0}, v_{0}\right)-\pi$ we get

$$
\begin{aligned}
d\left(\mathrm{x}_{\rho}^{-}(x, v), \mathrm{x}_{\rho}^{-}\left(x_{0}, v_{0}\right)\right) & =\pi-d\left(\mathrm{x}_{\rho}^{-}(x, v), \mathrm{x}_{\rho}^{+}\left(x_{0}, v_{0}\right)\right) \\
& <\pi-\left|\theta_{\rho}^{-}(x, v)-\theta_{\rho}^{+}\left(x_{0}, v_{0}\right)\right| \\
& =\pi-\left|\theta_{\rho}^{-}(x, v)-\theta_{\rho}^{-}\left(x_{0}, v_{0}\right)-\pi\right| \\
& \leq \theta_{\rho}^{-}(x, v)-\theta_{\rho}^{-}\left(x_{0}, v_{0}\right) .
\end{aligned}
$$

This means that $\tilde{\ell}_{\rho}^{-}(x, v)$ lies in the strict future of $\tilde{\ell}_{\rho}^{-}\left(x_{0}, v_{0}\right)$. Incorporating the case $(x, v)=\left(x_{0}, v_{0}\right)$ it follows that for every pair of points $p, q$ in the image $\tilde{\Lambda}_{\rho}^{-}$of $\tilde{\ell}_{\rho}^{-}$, $p$ lies in the causal future of $q$. By symmetry, we obtain reversely that $q$ lies in the future of $p$. It follows that $p=q$, i.e., that $\tilde{\ell}_{\rho}^{-}$takes only one value.

Similarly, one proves that $\tilde{\ell}_{\rho}^{+}$takes only one value. Hence, for every $(x, v)$ in $\mathrm{T}^{1} \tilde{\Sigma}, \ell_{\rho}^{-}(x, v)$ is the opposite $-\ell_{\rho}^{+}(x, v)$ of $\ell_{\rho}^{+}(x, v)$. This is impossible since there is a space-like geodesic between $\ell_{\rho}^{-}(x, v)$ and $\ell_{\rho}^{+}(x, v)$. This contradictions shows as required that $\ell_{\rho}^{+}(x, v)=\ell_{\rho}^{-} \circ \alpha(x, v)$.

Corollary 5.12. The mappings $\ell_{\rho}^{ \pm}$have the same image. They are homeomorphisms between $\partial \widetilde{\Sigma}$ and a topological acausal $(n-1)$-sphere $\Lambda_{\rho}$.

Proof. The equality of the images is an immediate consequence of Proposition 5.9. We only have to show that the mapping $\ell_{\rho}^{+}$(for example) is injective. Let $(x, v)$ and $(y, w)$ be two points of $\mathrm{T}^{1} \widetilde{\Sigma}$, belonging to two different stable leaves. Hence, there exists a point $(z, v)$ which is in $(x, v)$ 's stable leaf and $(y, w)$ 's unstable one. We thus have $\left.\left(\ell_{\rho}^{+}(x, v), \ell_{\rho}^{-}(\alpha(y, w))\right)=\left(\ell_{\rho}^{+}(z, v)\right), \ell_{\rho}^{-}(z, v)\right) \subseteq \mathcal{Y}$. In particular, $\ell_{\rho}^{+}(x, v)$ and $\ell_{\rho}^{-}(\alpha(y, w))=\ell_{\rho}^{+}(y, w)$ are joined by a space-like geodesic and must be different.

Proof of the first inclusion in Theorem 1.2. According to the last corollary, $\rho(\Gamma)$ preserves an acausal $(n-1)$-sphere. According to Lemma 5.11, $\rho$ is faithful. The only point left is to prove that $\rho(\Gamma)$ is a discrete subgroup of $\mathrm{SO}_{0}(2, n)$.

Assume this is not the case. Then there exist a sequence $\left(\gamma_{n}\right)_{(n \in \mathbb{N})}$ in $\Gamma$ such that $\left(\rho\left(\gamma_{n}\right)\right)_{(n \in \mathbb{N})}$ converges non-trivially to the identity. This sequence $\left(\gamma_{n}\right)_{(n \in \mathbb{N})}$ has to escape from any compact subset of $\Gamma$. Hence, by item (8) of $\S 5.1 .4$ one can assume, taking a subsequence if necessary, that there are two points $x^{+}, x^{-}$in $\partial \widetilde{\Sigma}$ such that 
$\left(\gamma_{n}\right)_{(n \in \mathbb{N})}$ converges uniformly on compact subsets of $\partial \widetilde{\Sigma} \backslash\left\{x^{-}\right\}$to the constant map taking the value $x^{+}$. This is in contradiction with the equivariance of the map $\ell_{\rho}^{+}$and the fact that the sequence $\left(\rho\left(\gamma_{n}\right)\right)_{(n \in \mathbb{N})}$ converges to the identity element.

\section{Dynamical properties}

From now on we consider a strictly GHC-regular representation $\rho: \Gamma \rightarrow \mathrm{SO}_{0}(2, n)$ with acausal limit set $\Lambda_{\rho}$, where $\Gamma$ is the fundamental group of a negatively curved Riemannian manifold $N$. In particular, and this will be essential for the proof, the group $\Gamma$ is hyperbolic in the sense of Gromov.

Let $\left(\gamma_{n}\right)_{(n \in \mathbb{N})}$ be a sequence in $\Gamma$ escaping to infinity. It will be convenient to consider the image sequence $\left(\rho\left(\gamma_{n}\right)\right)_{(n \in \mathbb{N})}$ as a sequence in $\mathrm{SO}_{0}(2, n+1)$ through the inclusion $\mathrm{SO}_{0}(2, n) \subset \mathrm{SO}_{0}(2, n+1)$ so that our dynamical study applies to $\operatorname{Ein}_{n+1}$ and hence to the $\rho(\Gamma)$-invariant conformal copy of $\operatorname{AdS}_{n+1}$ inside $\operatorname{Ein}_{n+1}$.

In [Fra05] (see also $\left[\mathrm{BCD}^{+} 08, \S 7\right]$ ), C. Frances studied the dynamical behavior in $\operatorname{Ein}_{n+1}$ of such a sequence $\left(\rho\left(\gamma_{n}\right)\right)_{(n \in \mathbb{N})}$. Taking subsequences if necessary, there are two possible behaviours (The ingredients of the proof are recalled afterwards):

(1) Balanced distortion: There are two light-like geodesics $\Delta^{+}, \Delta^{-}$in $\operatorname{Ein}_{n+1}$, called attracting and repelling photons, and two continuous mappings $\pi_{+}: \operatorname{Ein}_{n+1} \backslash$ $\Delta^{-} \rightarrow \Delta^{+}$and $\pi_{-}: \operatorname{Ein}_{n+1} \backslash \Delta^{+} \rightarrow \Delta^{-}$such that

- the fibers of $\pi_{+}$(respectively $\pi_{-}$) are past lightcones $C^{-}(x)$ of points in $\Delta^{-}$ (respectively of points in $\Delta^{+}$),

- for every compact subset $K \subset \operatorname{Ein}_{n+1} \backslash \Delta^{-}$, the sequence $\rho\left(\gamma_{n}\right)$ uniformly converges on $K$ to $\pi_{+}$,

- for every compact subset $K \subset \operatorname{Ein}_{n+1} \backslash \Delta^{+}$, the sequence $\rho\left(\gamma_{n}^{-1}\right)$ uniformly converges on $K$ to $\pi_{-}$.

(2) Unbalanced distortion: There are two points $x^{+}, x^{-}$in $\operatorname{Ein}_{n+1}$, called attracting and repelling poles, such that

- $\left\langle x^{+} \mid x^{-}\right\rangle \leq 0$,

- for every compact subset $K$ of $\operatorname{Ein}_{n+1}$ contained in $\Omega^{-}\left(x^{-}\right):=\left\{x \in \operatorname{Ein}_{n+1} \mid\right.$ $\left.\left\langle x \mid x^{-}\right\rangle<0\right\}$ (resp. $\Omega^{+}\left(x^{-}\right):=\left\{x \in \operatorname{Ein}_{n+1} \mid\left\langle x \mid x^{-}\right\rangle>0\right\}$ ) the sequence $\rho\left(\gamma_{n}\right)$ uniformly converges on $K$ to the constant map $x^{+}$(resp. $\left.-x^{+}\right)$,

- for every compact subset $K$ of $\operatorname{Ein}_{n+1}$ contained in $\Omega^{-}\left(x^{+}\right):=\left\{x \in \operatorname{Ein}_{n+1} \mid\right.$ $\left.\left\langle x \mid x^{+}\right\rangle<0\right\}$ (resp. $\Omega^{+}\left(x^{+}\right):=\left\{x \in \operatorname{Ein}_{n+1} \mid\left\langle x \mid x^{+}\right\rangle>0\right\}$ ) the sequence $\rho\left(\gamma_{n}^{-1}\right)$ uniformly converges on $K$ to the constant map $x^{-}$(resp. $-x^{-}$).

Remark 6.1. Our presentation differs from Frances' formulation. Indeed, in [Fra05] he defines the Einstein space as the projection of $\ell_{n+1}$ in the projective space $\mathbb{P}\left(\mathbb{R}^{n+3}\right)$, and not the projection in the sphere of rays $\mathbb{S}\left(\mathbb{R}^{n+3}\right)$. In other words, $\operatorname{Ein}_{n+1}$ is the double covering of the space he considers. Consequently, C. Frances 
had no need to distinguish future cones from past cones since they have the same projection in $\mathbb{P}\left(\mathbb{R}^{n+3}\right)$.

A nuisance of the option to consider the double covering is the non-uniqueness of pairs of attracting/repelling poles. Indeed, the opposite pair $\left(-x^{+},-x^{-}\right)$is also convenient. Moreover, if $\left\langle x^{-} \mid x^{+}\right\rangle=0$, we have four choices $\left( \pm x^{+}, \pm x^{-}\right)$of pairs of attracting/repelling poles.

Remark 6.2. Every element $\rho\left(\gamma_{n}\right)$ lies in the subgroup $\mathrm{SO}_{0}(2, n)$ of $\mathrm{SO}_{0}(2, n+1)$, i.e., it preserves the conformal embedding $\operatorname{AdS}_{n+1} \subset \operatorname{Ein}_{n+1}$ and its boundary $\partial \mathrm{AdS}_{n+1} \approx \operatorname{Ein}_{n}$. In that situation, all the limit objects $\Delta^{ \pm}, x^{ \pm}$involved in the various cases in the description of the asymptotic behavior of $\left(\rho\left(\gamma_{n_{k}}\right)\right)_{(k \in \mathbb{N})}$ are contained in this boundary. In particular, they avoid $\mathrm{AdS}_{n+1}$.

The dichotomy balanced/unbalanced is based on the Cartan decomposition of $\mathrm{SO}_{0}(2, n+1)$. More precisely, consider the following quadratic form on $\mathbb{R}^{n+3}$ :

$$
\mathfrak{q}_{2, n+1}:=-4 a_{1} b_{1}-4 a_{2} b_{2}+x_{1}^{2}+\cdots+x_{n-1}^{2} \text {. }
$$

Observe that the spaces $\left(\mathbb{R}^{n+3}, \mathfrak{q}_{2, n+1}\right)$ and $\left(\mathbb{R}^{2, n+1}, \mathrm{q}_{2, n+1}\right)$ are isometric, the isometry being given by the map

$$
\begin{aligned}
& \left(a_{1}, b_{1}, a_{2}, b_{2}, x_{1}, \ldots, x_{n-1}\right) \\
& \quad \rightarrow\left(\left(a_{1}+b_{1}\right) / 2,\left(a_{2}+b_{2}\right) / 2, x_{1}, \ldots, x_{n-1},\left(a_{1}-b_{1}\right) / 2,\left(a_{2}-b_{2}\right) / 2\right) .
\end{aligned}
$$

Let $\mathfrak{U}$ be the free abelian subgroup of rank 2 of $\mathrm{SO}_{0}(2, n+1)$ made of elements $a(\lambda, \mu)$ whose matrix in the coordinates $\left(a_{1}, a_{2}, b_{1}, b_{2}, x_{1}, \ldots, x_{n-1}\right)$ is diagonal with components $\exp (\lambda), \exp (\mu), \exp (-\lambda), \exp (-\mu), 1, \ldots, 1$. This defines a real split Cartan subgroup of $\mathrm{SO}_{0}(2, n+1)$, and we consider the Weyl chamber $\mathfrak{U}^{+} \subset \mathfrak{U}$ containing the elements $a(\lambda, \mu)$ such that $0 \leq \mu \leq \lambda$. The Cartan decomposition theorem ensures that every $\rho\left(\gamma_{n}\right)$ can be written in the form $\rho\left(\gamma_{n}\right)=\mathfrak{F}_{n} a_{n} \mathfrak{l}_{n}$ such that $a_{n}=a\left(\lambda_{n}, \mu_{n}\right)$ belongs to $\mathfrak{U}^{+}$and $\mathfrak{k}_{n}, \mathfrak{l}_{n}$ belong to the stabilizer $\mathfrak{K}$ of the negative definite 2-plane

$$
\left\{a_{1}=b_{1}, a_{2}=b_{2}, x_{1}=\cdots=x_{n-1}=0\right\}
$$

Note that this stabilizer is a maximal compact subgroup. Observe that elements of $\Omega$ are isometries of the Euclidean norm $\left\|\left(u, v, x_{1}, \ldots, x_{n}\right)\right\|_{0}^{2}:=u^{2}+v^{2}+x_{1}^{2}+\cdots+x_{n}^{2}$. Since $\left(\rho\left(\gamma_{n}\right)\right)_{(n \in \mathbb{N})}$ escapes from any compact set, the sequence $\left(\lambda_{n}\right)_{n \in \mathbb{N})}$ is not bounded from above. By compactness of $\Omega$ there is a converging subsequence, i.e., a subsequence $\left(\gamma_{n_{k}}\right)_{(k \in \mathbb{N})}$ such that $\mathfrak{k}_{n_{k}}, \mathfrak{l}_{n_{k}}$ converge to some elements $\mathfrak{k}_{\infty}, \mathfrak{l}_{\infty}$ of $\Re$, and such that $\lambda_{n_{k}}$ converges to $+\infty$, while the difference $\exp \left(\mu_{n_{k}}-\lambda_{n_{k}}\right)$ converges to a number $v \in[0,1]$. 
6.1. Balanced distortion. Balanced distortion occurs when $v>0$. Denote by $P^{-}$, $P^{+}$the codimension two subspaces $\left\{a_{1}=a_{2}=0\right\}$ and $\left\{b_{1}=b_{2}=0\right\}$ respectively. Consider the following linear endomorphisms of $\mathbb{R}^{n+3}$ :

$$
\begin{aligned}
& \Pi_{0}^{+}\left(a_{1}, b_{1}, a_{2}, b_{2}, x_{1}, \ldots, x_{n-1}\right)=\left(a_{1}, 0, v a_{2}, 0, \ldots, 0\right), \\
& \Pi_{0}^{-}\left(a_{1}, b_{1}, a_{2}, b_{2}, x_{1}, \ldots, x_{n-1}\right)=\left(0, b_{1}, 0, v b_{2}, 0, \ldots, 0\right) .
\end{aligned}
$$

They induce two maps $\pi_{0}^{+}: \mathbb{S}\left(\mathbb{R}^{n+3}\right) \backslash \mathbb{S}\left(P^{-}\right)$and $\pi_{0}^{-}: \mathbb{S}\left(\mathbb{R}^{n+3}\right) \backslash \mathbb{S}\left(P^{+}\right)$. Clearly, as a sequence of transformations of $\mathbb{S}\left(\mathbb{R}^{n+3}\right),\left(a_{n_{k}}\right)_{(k \in \mathbb{N})}$ converges uniformly on compact subsets of $\mathbb{S}\left(\mathbb{R}^{n+3}\right) \backslash \mathbb{S}\left(P^{-}\right)$to the map induced by $\pi_{0}^{+}$, and a similar remark applies to the inverse sequence $\left(a_{n_{k}}^{-1}\right)_{(k \in \mathbb{N})}$. It follows that the sequence $\left(\rho\left(\gamma_{n_{k}}\right)\right)_{(n k \in \mathbb{N})}$ converges uniformly on compact subsets of $\mathbb{S}\left(\mathbb{R}^{n+3}\right) \backslash \mathbb{S}\left(\mathfrak{l}_{\infty}^{-1} P^{-}\right)$to $\mathfrak{k}_{\infty} \circ \pi_{0}^{+} \circ \mathfrak{I}_{\infty}$ and that $\left(\rho\left(\gamma_{n_{k}}^{-1}\right)\right)_{(n k \in \mathbb{N})}$ converges uniformly on compact subsets of $\mathbb{S}\left(\mathbb{R}^{n+3}\right) \backslash \mathbb{S}\left(\mathfrak{F}_{\infty} P^{+}\right)$to $\mathfrak{I}_{\infty}^{-1} \circ \pi_{0}^{-} \circ \mathfrak{k}_{\infty}^{-1}$. The description of the dynamic in $\operatorname{Ein}_{n+1}$ given above follows by observing that the intersections $P^{ \pm} \cap \mathcal{C}_{n+1}$ are isotropic 2-planes.

6.2. Unbalanced distortion. This corresponds to the case $v=0$. Identify the sphere $\mathbb{S}\left(\mathbb{R}^{n+3}\right)$ of rays with the $\|\cdot\|_{0}$-unit sphere. The attracting fixed points of the action of $a_{n}$ in $\mathbb{S}\left(\mathbb{R}^{n+3}\right)$ are $\pm x_{0}^{+}$where $x_{0}^{+}=(1,0, \ldots, 0)$, and the repelling fixed points are $\pm x_{0}^{-}$where $x_{0}^{-}=(0,0,1,0, \ldots, 0)$. Observe that the $\mathfrak{q}_{2, n+1}$-orthogonal of the vector $x_{0}^{+}$is the hyperplane $\left\{b_{1}=0\right\}$. This hyperplane is also the orthogonal of $x_{0}^{-}$for the Euclidean norm $\|\cdot\|_{0}$. Similarly, $\left(x_{0}^{-}\right)^{\perp}=\left\{b_{2}=0\right\}$ is the $\|\cdot\|_{0}$-orthogonal of $x_{0}^{+}$.

For every $\varepsilon>0$ let $C_{0}^{-}(\varepsilon)$ be the spherical ball in $\mathbb{S}\left(\mathbb{R}^{n+3}\right)$ of radius $\pi / 2-\varepsilon$ centered at $x_{0}^{+}$. It can also be defined as the connected component containing $x_{0}^{+}$of the complement in $\mathbb{S}\left(\mathbb{R}^{n+3}\right)$ of the $\varepsilon$-neighborhood of $\left(x_{0}^{-}\right)^{\perp}$.

Every vector in $\mathbb{R}^{n+3}$ splits as a sum $r x_{0}^{+}+y$ with $y$ in $\left(x_{0}^{-}\right)^{\perp}$. Under the action of $a\left(\lambda_{n}, \mu_{n}\right)$ the component $r x_{0}^{+}$is multiplied by $\exp \left(\lambda_{n}\right)$ whereas the norm of the component $y$ is multiplied by at most $\exp \left(\mu_{n}\right)$. One easily obtains:

Lemma 6.3. Let $a\left(\lambda_{n}, \mu_{n}\right)$ be a sequence in $\mathfrak{A}^{+}$with no balanced distortion. For any $\varepsilon>0$ and any $\eta>0$ there is $N>0$ such that, for every $n>N$, the restriction of $a\left(\lambda_{n}, \mu_{n}\right)$ to $C_{0}^{-}(\varepsilon)$ is $\eta$-Lipschitz, with image contained in $C_{0}^{+}(\pi-\eta)$.

The description of the dynamic of unbalanced converging subsequences on $\operatorname{Ein}_{n+1}$ given above follows easily. The attracting pole $x^{+}$is simply the image of $x_{0}^{+}$by $\mathfrak{k}_{\infty}$, and the repelling pole is $x^{-}=\mathfrak{l}_{\infty}^{-1} x_{0}^{-}$. We entered in such detail that the next lemma is now obvious. Consider the hemisphere $\mathbb{D}^{-}=\left\{x \in \mathbb{S}\left(\mathbb{R}^{n+3}\right) \mid\left\langle x \mid x^{-}\right\rangle<0\right\}$. For every $\varepsilon>0$ let $C^{-}(\varepsilon)$ be the set of points in $D^{-}$at distance at least $\varepsilon$ from $\left(x^{-}\right)^{\perp} \cap \mathbb{S}\left(\mathbb{R}^{n+3}\right)$. Since $\mathfrak{F}_{n}, \mathfrak{l}_{n}$ are isometries for $\|\cdot\|_{0}$, we have: 
Lemma 6.4. For any $\varepsilon>0$ and any $\eta>0$ there is $N>0$ such that, for every $k>N$, the restriction of $\rho\left(\gamma_{n_{k}}\right)$ to $C^{-}(\varepsilon)$ is $\eta$-Lipschitz, with image contained in the ball centered at $x^{+}$and of radius $\eta$.

The statement we actually need is:

Corollary 6.5. Assume that $x^{+}$belongs to $\rho\left(\gamma_{n_{k}}\right) D^{-}$for $k$ sufficiently large. Then the differential at $x^{+}$of the inverse of $\rho\left(\gamma_{n_{k}}\right)$, as a transformation of the unit sphere $\mathbb{S}\left(\mathbb{R}^{2, n+1}\right)$, expands all the vectors tangent to the sphere at $x^{+}$by at least a factor $v_{k}$ such that $v_{k} \rightarrow+\infty$ when $k \rightarrow+\infty$.

Balanced distortion is the typical behavior of converging subsequences $\left(\rho\left(\gamma_{n_{k}}\right)\right)_{(k \in \mathbb{N})}$ when $\rho(\Gamma)$ acts properly discontinuously on $\operatorname{AdS}_{n+1}$. But our situation here is different: by hypothesis, the group $\rho(\Gamma)$ preserves an acausal limit set $\Lambda_{\rho}$ which is not purely light-like since $E\left(\Lambda_{\rho}\right) \neq \emptyset$ (Lemma 3.6).

Proposition 6.6. No sequence in $\rho(\Gamma)$ has balanced distortion.

Proof. Assume, on the contrary, that some sequence $\left(\rho\left(\gamma_{n}\right)\right)_{(n \in \mathbb{N})}$ has balanced distortion. Denote by $\Delta^{ \pm}$the repelling and attracting photons. Since $\Lambda_{\rho}$ is an acausal topological sphere, it intersects $\Delta^{+}$at an unique point $x^{+}$Since $\Lambda_{\rho}$ is $\rho(\Gamma)$-invariant, the image by $\pi^{+}$of $\Lambda_{\rho} \backslash \Delta^{-}$is $x^{+}$. The fibers of $\pi^{+}$are past cones of elements of $\Delta^{-}$. Hence, $\Lambda_{\rho} \backslash \Delta^{-}$is contained in the past cone $C^{-}\left(x^{+}\right)$Since $\Delta^{-} \cap \Lambda_{\rho}$ is a compact embedded segment, $\Lambda_{\rho} \backslash \Delta^{-}$is dense in $\Lambda_{\rho}$ (this argument is correct when the dimension of $\Lambda_{\rho}$ is $\geq 2$. For the case where $\Lambda_{\rho}$ is a topological circle, see [Mes07], [ABB $\left.{ }^{+} 07\right]$, or [BBZ07], $\left.\S 6.2\right)$. Hence $\Lambda_{\rho}$ is contained in $C^{-}\left(x^{+}\right)$. It is impossible since $\Lambda_{\rho}$ is not purely light-like.

Remark 6.7. The ambiguity in the definition of pairs of attracting/repelling poles, mentioned in Remark 6.1, can be removed for GHC-regular representations by selection the poles that belong to $\Lambda_{\rho}$. Indeed:

$\Lambda_{\rho}$ contains an attracting pole. Since it is not contained in a cone $C\left( \pm x^{-}\right), \Lambda_{\rho}$ intersects $\Omega^{+}\left(x^{-}\right)$or $\Omega^{-}\left(x^{-}\right)$, and the $\rho\left(\gamma_{n}\right)$-orbit of a point in this intersection accumulates in $\pm x^{+}$, which therefore belongs to $\Lambda_{\rho}$. Similarly, $\Lambda_{\rho}$ contains a repelling pole.

$\Lambda_{\rho}$ contains one and only one attracting pole. For $x^{+}$and $-x^{+}$cannot both belong to $\Lambda_{\rho}$ since $\Lambda_{\rho}$ is not purely light-like. Similarly, $\Lambda_{\rho}$ contains one and only one repelling pole.

Observe that the condition $\left\langle x^{-} \mid x^{+}\right\rangle \leq 0$ is fulfilled since $\Lambda_{\rho}$ is achronal.

\section{Convex hull of GHC-representations}

7.1. Metric on the convex hull. In the sequel we need to define a $\rho(\Gamma)$-metric on $\operatorname{Conv}\left(\Lambda_{\rho}\right)$. Since the action is cocompact, all these metrics are quasi-isometric to 
each other (see $\S 7.3$ ) and the choice is not important. However, in order to sustain our argumentation, we choose a specific metric.

Let $\Omega$ be a bounded open domain in $\mathbb{P}\left(\mathbb{R}^{n}\right)$, i.e., an open domain contained in an affine chart and such that the closure $\bar{\Omega}$ in this affine chart is compact. The Hilbert distance between two points $x, y$ in $\Omega$ is

$$
d^{H}(x, y):=\log (a, b, x, y),
$$

where $a, b$ are the two intersections between $\partial \Omega$ and the projective line containing $x$ and $y$, and where $(a, b, x, y)$ is the cross-ratio. This is a distance function, and the associated metric is proper, geodesic, and every projective transformation preserving $\Omega$ preserves the Hilbert distance of points. Moreover, the geodesics for this metrics are intersections between projective lines and $\Omega$ (see [BK53]).

The interior of the convex hull $\operatorname{Conv}\left(\Lambda_{\rho}\right)$ is a bounded open domain, hence admits a well-defined $\rho(\Gamma)$-invariant Hilbert metric. However, in the sequel we will need metrics defined on $\operatorname{Conv}\left(\Lambda_{\rho}\right) \backslash \Lambda_{\rho}$ and not only on its interior. Hence we have to enlarge $\operatorname{Conv}\left(\Lambda_{\rho}\right)$ to another convex domain, still bounded and $\rho(\Gamma)$-invariant, but containing the boundaries $\widetilde{S}_{\rho}^{ \pm}$.

A suitable solution is to consider, for $\varepsilon>0$ small enough, the domain $\operatorname{Conv}\left(\Lambda_{\rho}\right)_{\varepsilon}$ in $\operatorname{AdS}_{n+1}$ made of points $x$ such that every causal curve in $\operatorname{AdS}_{n+1}$ joining $x$ to an element of $\operatorname{Conv}\left(\Lambda_{\rho}\right)$ is of Lorentzian length $\leq \varepsilon$. It follows quite easily from the compactness of $\rho(\Gamma) \backslash \operatorname{Conv}\left(\Lambda_{\rho}\right)$ that for $\varepsilon$ small enough $\operatorname{Conv}\left(\Lambda_{\rho}\right)_{\varepsilon}$ is contained in $E\left(\Lambda_{\rho}\right)$. The proof that $\operatorname{Conv}\left(\Lambda_{\rho}\right)_{\varepsilon}$ is still convex is straightforward, we refer to [BBZ07], Proposition 6.31, for a proof formulated in dimension $2+1$, but valid in any dimension. Observe also that $\operatorname{Conv}\left(\Lambda_{\rho}\right)_{\varepsilon}$ is still bounded, and that its interior contains $\operatorname{Conv}\left(\Lambda_{\rho}\right) \backslash \Lambda_{\rho}$.

In the sequel, we fix, once for all, $\varepsilon$ and denote by $d^{H}$ the restriction to $\operatorname{Conv}\left(\Lambda_{\rho}\right)$ of the Hilbert metric of $\operatorname{Conv}\left(\Lambda_{\rho}\right)_{\varepsilon}$.

Remark 7.1. If ] $a, b$ [ is a space-like geodesic joining two points in $\operatorname{Ein}_{n}$, then for any $x, y$ in ] $a, b$ [ the AdS-length of the piece of geodesic between $x$ and $y$ is $\log (a, b, x, y)$. This is a generalization of the well-known fact that the Hilbert metric on the Klein model of the hyperbolic space is isometric to the hyperbolic metric, see e.g. [Sa199], Theorem 2.2.1.11. It follows that in the case where $a, b$ lies on $\Lambda_{\rho}$ this length is the Hilbert distance $d^{H}(x, y)$.

7.2. Dirichlet domains. If $\Gamma$ acts freely and properly discontinuously on a proper complete metric space $X$, there is a well-known way to construct a fundamental domain of its action: the Dirichlet domain (see [Rat06], pp. 243-245). Here the action we consider does not preserve a Riemannian metric, but the construction of Dirichlet domain extends easily to our situation:

Definition 7.2. Fix an element $x_{0}$ of $\operatorname{Conv}\left(\Lambda_{\rho}\right)$. For every $\gamma$ in $\Gamma$, let $D(\gamma)$ be the domain $\left\{x \in E\left(\Lambda_{\rho}\right) \mid\left\langle x \mid x_{0}\right\rangle>\left\langle x \mid \rho(\gamma) x_{0}\right\rangle\right\}$, considering $E\left(\Lambda_{\rho}\right)$ as a subset of $\left.\operatorname{AdS}_{n+1} \subset \mathbb{R}^{2, n}\right)$. The Dirichlet domain $D(\Gamma)$ is the intersection $\bigcap_{\gamma \in \Gamma} D(\gamma)$. 
Remark 7.3. Since the quotient $M$ is globally hyperbolic, it admits no closed causal curve. Thus $x_{0}$ and $\rho(\gamma) x_{0}$ are not causally related: the $\mathrm{q}_{2, n}$-norm of $\left(\rho(\gamma) x_{0}-x_{0}\right)$ is positive. The domain $D(\gamma)$ is the interior of the intersection between $E\left(\Lambda_{\rho}\right)$ and the half AdS-space $\mathrm{H}^{-}\left(\rho(\gamma) x_{0}-x_{0}\right)$.

Lemma 7.4. The complements $H(\gamma)=E\left(\Lambda_{\rho}\right) \backslash D(\gamma)$ form a locally finite family of subsets of $E\left(\Lambda_{\rho}\right)$.

Proof. Assume by contradiction that a compact set $K$ of $E\left(\Lambda_{\rho}\right)$ intersects infinitely many such sets $\left(H\left(\gamma_{n}\right)\right)_{n \geq 0}$. According to Proposition 6.6 and Remark 6.7 we can assume, taking a subsequence if necessary, that the map induced in the Klein model by $\rho\left(\gamma_{n}^{-1}\right)$ converges uniformly on $K$ to a point $x^{-}$in $\Lambda_{\rho}$.

On the other hand, there is a sequence of points $\left(x_{n}\right)_{(n \in \mathbb{N})}$ in $K$, converging to a point $x$, and such that

$$
\left\langle x_{n} \mid x_{0}\right\rangle \leq\left\langle x_{n} \mid \rho\left(\gamma_{n}\right) x_{0}\right\rangle=\left\langle\rho\left(\gamma_{n}^{-1}\right) x_{n} \mid x_{0}\right\rangle
$$

for every $n$. Since the $\rho\left(\gamma_{n}^{-1}\right) x_{n}$ has $\mathrm{q}_{2, n}$-norm -1 , the convergence in the Klein model towards $x^{-}$means that for some sequence $\lambda_{n} \rightarrow 0$ the $\lambda_{n} \rho\left(\gamma_{n}^{-1}\right)\left(x_{n}\right)$ converges to a representant $\hat{x}^{-}$in $\ell_{n}$ of $x^{-}$. Hence

$$
\left\langle x_{n} \mid x_{0}\right\rangle \leq \frac{1}{\lambda_{n}}\left\langle x_{0} \mid \lambda_{n} \rho\left(\gamma_{n}^{-1}\right) x_{n}\right\rangle .
$$

The left term converges to $\left\langle x \mid x_{0}\right\rangle$, and since $\frac{1}{\lambda_{n}}$ converges to $+\infty$ and $\left\langle x_{0} \mid \lambda_{n} \rho\left(\gamma_{n}^{-1}\right) x_{0}\right\rangle$ converges to the negative number $\left\langle x_{0} \mid \hat{x}^{-}\right\rangle$, the right term converges to $-\infty$. This is a contradiction.

A first corollary of this lemma is that $D(\Gamma)$ is open, and its closure $\operatorname{Cl}(D(\Gamma))$ is the intersection of the closures of the $D(\gamma)$.

Lemma 7.5. The $\Gamma$-iterates of $\mathrm{Cl}(D(\Gamma))$ cover $E\left(\Lambda_{\rho}\right)$, i.e.,

$$
E\left(\Lambda_{\rho}\right)=, \bigcup_{\gamma \in \Gamma} \rho(\gamma) \operatorname{Cl}(D(\Gamma)) .
$$

Proof. Let $x \in E\left(\Lambda_{\rho}\right)$; consider the map $\xi: \Gamma \rightarrow \mathbb{R}$ defined by $\xi(\gamma)=\left\langle x \mid \rho(\gamma) x_{0}\right\rangle$. If there is a sequence $\gamma_{n}$ such that $\xi\left(\gamma_{n}\right)$ increases, the argument used in the proof above with the constant sequence $x_{n}=x$ leads to a contradiction. Hence $\xi$ attains its maximum at some $\gamma_{0}$, i.e., $\left\langle\rho\left(\gamma_{0}\right) x_{0} \mid x\right\rangle \geq\left\langle\rho(\gamma) x_{0} \mid x\right\rangle$ for every $\gamma$ in $\Gamma$. It follows that $\rho\left(\gamma_{0}\right)^{-1} x$ belongs to $\mathrm{Cl}(D(\Gamma))$.

Lemma 7.6. The iterates $\rho(\gamma) D(\Gamma)$ are disjoint from each other.

Proof. If $x$ lies in $\rho(\gamma) D(\Gamma)$, then for every $h$ in $\rho(\Gamma) \backslash \rho(\gamma)$ we have

$$
\left\langle x \mid \rho(\gamma) x_{0}\right\rangle>\left\langle x \mid h x_{0}\right\rangle .
$$


If moreover $x$ lies in $\rho\left(\gamma^{\prime}\right) D(\Gamma)$ with $\rho\left(\gamma^{\prime}\right) \neq \rho(\gamma)$ we have

$$
\left\langle x \mid \rho\left(\gamma^{\prime}\right) x_{0}\right\rangle>\left\langle x \mid \rho(\gamma) x_{0}\right\rangle .
$$

We obtain a contradiction to the above in the case $h=\rho\left(\gamma^{\prime}\right)$.

The two lemmas above show that $\mathrm{Cl}(D(\Gamma))$ is a fundamental domain for the action of $\rho(\Gamma)$ on $E\left(\Lambda_{\rho}\right)$. From now on we restrict to the intersection $\mathrm{Cl}(D(\Gamma)) \cap \operatorname{Conv}\left(\Lambda_{\rho}\right)$ and denote it by $\bar{D}_{\text {conv }}(\Gamma)$. Since the quotient $C(M)=\rho(\Gamma) \backslash \operatorname{Conv}\left(\Lambda_{\rho}\right)$ is compact, we have:

Proposition 7.7. $\bar{D}_{\text {conv }}(\Gamma)$ is a compact fundamental domain for the action of $\rho(\Gamma)$ on $\operatorname{Conv}\left(\Lambda_{\rho}\right)$.

This compactness property implies that $\bar{D}_{\text {conv }}(\Gamma)$ is the intersection between the convex hull and a finite-sided convex polyhedron. Hence $\bar{D}_{\text {conv }}(\Gamma)$ itself is also convex.

7.3. Quasi-isometry between the group and the convex hull. A map $f: X \rightarrow X^{\prime}$ between two metric spaces $(X, d),\left(X^{\prime}, d^{\prime}\right)$ is a quasi-isometry if there exist $a>0$, $b>0$ such that

$$
(1 / a) d(x, y)-b<d^{\prime}(f(x), f(y))<a d(x, y)+b,
$$

and such that any point in $X^{\prime}$ is at distance at most $b$ from the image of $f$.

Now consider the set $S$ of elements $\gamma$ of $\Gamma$ such that the intersection between $\rho(\gamma) \bar{D}_{\text {conv }}(\Gamma)$ and $\bar{D}_{\text {conv }}(\Gamma)$ is non-empty. According to Lemma 7.4, this set is finite, and according to Lemmas 7.5, 7.6 it is a generating set of $\Gamma$. We consider the Cayley graph $\left(\Gamma_{S}, d_{S}\right)$, i.e., the simplicial metric space having for vertices the elements of $\Gamma$, and where two vertices $\gamma, \gamma^{\prime}$ are connected by an edge of length 1 if and only if $\gamma^{\prime} \gamma^{-1}$ lies in $S$.

Since $\Gamma$ acts cocompactly on $\operatorname{Conv}\left(\Lambda_{\rho}\right)$, the map

$$
\hat{\jmath}:\left(\Gamma_{S}, d_{S}\right) \rightarrow\left(\operatorname{Conv}\left(\Lambda_{\rho}\right), d^{H}\right)
$$

that associates to any vertex $\gamma$ the element $\rho(\gamma) x_{0}$ of $\rho(\gamma) \bar{D}_{\text {conv }}(\Gamma)$ is a quasi-isometry.

Recall that the group $\Gamma$ we consider is Gromov-hyperbolic. For the notions and properties of hyperbolic spaces or groups, we refer to [Gro], [GdLH $\left.{ }^{+} 90\right]$. By definition, the Gromov boundary of a hyperbolic geodesic space $(X, d)$ is the space of complete geodesic rays modulo the equivalence relation identifying two rays staying at bounded distance one from the other. Any quasi-isometry between hyperbolic spaces extends as a homeomorphism between their Gromov boundary: the image by a quasi-isometry of a geodesic ray is quasi-geodesic, i.e., a map $c:[0,+\infty[\rightarrow X$ such that

$$
1 / a|t-s|-b \leq d(c(t), c(s)) \leq a|s-t|+b
$$


for some $a, b>0$. Moreover, for every $a, b>0$, there is a constant $D$ such that for every $(a, b)$-quasi-geodesic ray $c:[0,+\infty[\rightarrow X$ there is a geodesic ray $c_{0}:\left[0,+\infty\left[\rightarrow X\right.\right.$ such that, for every $t$, the distance $c(t)$ to the image of $c_{0}$ is less than $D$, and the distance of $c_{0}(t)$ to the image of $c$ is less than $D$. We say that $c$ is at bounded distance $\leq D$ from $c_{0}$.

It follows that the quasi-isometry between $\Gamma_{S}$ and $\widetilde{\Sigma}$ extends to a homeomorphism between $\partial \Gamma$ and the conformal sphere $\partial \widetilde{\Sigma}$.

Proposition 7.8. The map $\hat{\jmath}$ extends as a homeomorphism $\mathrm{J}$ between the Gromov boundary $\partial \Gamma \approx \partial \widetilde{\Sigma}$ and the limit set $\Lambda_{\rho}$.

Proof. Let $\left(\gamma_{n}\right)_{(n \in \mathbb{N})}$ be the sequence of vertices of $\Gamma_{S}$ visited by a complete geodesic ray $r_{0}$ in $\left(\Gamma_{S}, d_{S}\right)$. According to the above discussion, there exists a constant $D \geq 0$ such that the image $\hat{\jmath}\left(r_{0}\right)$ is at bounded $d^{H}$-distance $D$ from a geodesic ray in $\left(\operatorname{Conv}\left(\Lambda_{\rho}\right), d^{H}\right)$, i.e., a projective segment $\left[x, y^{+}\left[\right.\right.$where $x$ lies in $\operatorname{Conv}\left(\Lambda_{\rho}\right)$ and $y^{+}$an element in $\partial \operatorname{Conv}\left(\Lambda_{\rho}\right)_{\varepsilon}$. Since this geodesic ray, of infinite $d^{H}$-length, is contained in $\operatorname{Conv}\left(\Lambda_{\rho}\right)$ the limit point $y^{+}$has to lie in $\Lambda_{\rho}$.

On the other hand, according to Proposition 6.6 every subsequence of $\left(\gamma_{n}\right)_{(n \in \mathbb{N})}$ admits a subsequence $\left(\gamma_{n_{k}}\right)_{(k \in \mathbb{N})}$ with mixed or bounded distortion: there is an attracting pole $x^{+}$in $\Lambda_{\rho}$ such that $\left(\rho\left(\gamma_{n_{k}}\right)\right)_{(k \in \mathbb{N})}$ converges uniformly on compact subsets of $E\left(\Lambda_{\rho}\right)$ to the constant map $x^{+}$. In particular, $x_{k}=\hat{\jmath}\left(\gamma_{n_{k}}\right)=\rho\left(\gamma_{n_{k}}\right)\left(x_{0}\right)$ converges to $x^{+}$.

If $x^{+} \neq y^{+}$then $] x^{+}, y^{+}\left[\right.$is a complete geodesic in $\left(\operatorname{Conv}\left(\Lambda_{\rho}\right), d^{H}\right)$ of infinite length. Hence there is a complete geodesic $c$ in $\Gamma_{S}$ such that $\hat{j}(c)$ is a quasi-geodesic at bounded distance from $] x^{+}, y^{+}\left[\right.$. Therefore the geodesic ray $r_{0}$ alternatively approximates both ends of $c$. This is not possible, since these ends are distinct and a geodesic ray admits only one accumulation point in $\partial \Gamma$.

Therefore $x^{+}=y^{+}$. It follows that $x^{+}$does not depend on the subsequence, and that $y^{+}$is the extremity of any $d^{H}$-geodesic ray at bounded distance from $\hat{\jmath}\left(r_{0}\right)$. Hence the map $J:\left[r_{0}\right] \in \partial \Gamma \rightarrow x^{+} \in \Lambda_{\rho}$ is well defined.

We now prove the continuity of $J$. Let $V$ be a neighborhood of $x^{+}$in $\Lambda_{\rho}$. Let $U$ be a neighborhood of $x^{+}$in $\operatorname{Ein}_{n+1}$ disjoint from $x_{0}$, such that $U \cap \Lambda_{\rho} \subset V$ and that $U \cap \operatorname{AdS}_{n+1}$ is convex. Finally, let $\mathfrak{H}^{+}(v)$ be a convex cap contained in $U$ such that $x^{+}$is in the interior of the topological disk $\mathfrak{H}^{+}(v) \cap \partial \operatorname{Conv}\left(\Lambda_{\rho}\right)$. The geodesic segment $\left[x_{0}, x^{+}\left[\right.\right.$crosses $\mathfrak{S}(v)$ at some point $x_{1}$. Let $x_{2}$ be another point of that segment sufficiently close to $x^{+}$so that the Hilbert distance between $x_{2}$ and $\mathfrak{H}^{-}(v)$ is bigger that $2 D$, where $D$ is the constant such that for every geodesic ray in $\Gamma_{S}$ there is a $d^{H}$-geodesic in $\operatorname{Conv}\left(\Lambda_{\rho}\right)$ at uniform distance $D$ from $\hat{\jmath}(r)$.

The point $x_{2}$ is at distance $D$ from an element $\rho\left(\gamma_{k}\right) x_{0}$ of $\hat{j}\left(r_{0}\right)$. Let $W$ be the neighborhood of $\left[r_{0}\right]$ made of equivalence classes $[r]$ of geodesic rays $r$ starting from the identity and containing $\gamma_{k}$. Then, $\hat{j}(r)$ is at bounded distance $D$ from the geodesic segment $\left[x_{0}, J([r])\left[\right.\right.$. Hence $\left[x_{0}, J([r])\left[\right.\right.$ contains a point $y_{2}$ at distance $\leq D$ from $\rho\left(\gamma_{k}\right) x_{0}$, hence at distance $\leq 2 D$ from $x_{2}$. According to our choice of $x_{2}$, this point 
$y_{2}$ lies on the same side of the wall $\mathfrak{S}(v)$ as $x_{2}$, i.e., in $\mathfrak{H}^{+}(v)$. Hence $\left[x_{0}, J([r])[\right.$ crosses $\mathfrak{S}(v)$ before reaching $J([r])$. Since $U$ is convex, it follows that $J([r])$ lies in $U$, hence, in $V$. The continuity of $\hat{j}$ is proved.

If $[r]$ and $\left[r^{\prime}\right]$ are two distinct elements in $\partial \Gamma$, there exists a complete geodesic $c: \mathbb{R} \rightarrow \Gamma$ asymptotic to $r$ near $-\infty$ and to $r^{\prime}$ near $+\infty$. The quasi-geodesic $\hat{\jmath}(c)$ is at bounded distance from a geodesic $] J([r]), J\left(\left[r^{\prime}\right]\right)\left[\right.$ in $\operatorname{Conv}\left(\Lambda_{\rho}\right)$. It follows that $J([r]) \neq J\left(\left[r^{\prime}\right]\right)$, and the map is injective.

Finally, for any $x$ in $\Lambda_{\rho}$, the $d^{H}$-geodesic ray $\left[x_{0}, x[\right.$ is at bounded distance from the image by $\hat{\jmath}$ of a quasi-geodesic ray in $\Gamma_{S}$, hence from the image by $\hat{j}$ of a geodesic ray. It follows that $J$ is onto. Since $\partial \Gamma$ is compact, the bijective map $J$ is an homeomorphism. The proposition is proved.

Remark 7.9. It was convenient for the proof above to consider $\left(\Gamma_{S}, d_{S}\right)$. But this metric space is quasi-isometric in a $\Gamma$-equivariant way to $\widetilde{\Sigma}$ and also $\mathrm{T}^{1} \widetilde{\Sigma}$. Hence, a corollary of Proposition 7.8 is that any quasi-isometry $\hat{\jmath}_{c}: \mathrm{T}^{1} \widetilde{\Sigma} \rightarrow \operatorname{Conv}\left(\Lambda_{\rho}\right)$ extends as a homeomorphism $J_{c}$ between the Gromov boundary $\partial \mathrm{T}^{1} \widetilde{\Sigma}$ and $\Lambda_{\rho}$.

\subsection{The geodesic flow of the GHC-regular spacetime}

Definition 7.10. The non-wandering subset, denoted by $\mathcal{N}\left(\Lambda_{\rho}\right)$, is the subset of $\varepsilon^{1} \operatorname{AdS}_{n+1}$ made of vectors $(x, v)$ such that $\ell^{ \pm}(x, v)$ lie in $\Lambda_{\rho}$. The geodesic flow on $\mathcal{N}\left(\Lambda_{\rho}\right)$ is the flow $\widetilde{\Phi}_{\mathcal{N}}^{t}$ defined by $\widetilde{\Phi}_{\mathcal{N}^{t}}^{t}(x, v)=\left(x^{t}, v^{t}\right)$, where $x^{t}$ is the point on the geodesic tangent to $(x, v)$ at distance $t$ (along the geodesic) from $x$, and $v^{t}$ the vector tangent at $x^{t}$ to this geodesic.

This definition is $\rho(\Gamma)$-equivariant, and we denote by $\mathcal{N}(\rho)$ the quotient of $\mathcal{N}\left(\Lambda_{\rho}\right)$ by $\rho(\Gamma)$ and $\Phi_{\mathcal{N}}^{t}$ the flow on $\mathcal{N}(\rho)$ induce by $\widetilde{\Phi}_{\mathcal{N}}^{t}$.

The projection of $\mathcal{N}\left(\Lambda_{\rho}\right)$ in $\operatorname{AdS}_{n+1}$ is obviously contained in the convex hull $\operatorname{Conv}\left(\Lambda_{\rho}\right)$. Since $\ell^{ \pm}$is continuous, and since $\Lambda_{\rho}$ and $C(M)$ are compact sets, the quotient $\mathcal{N}(\rho)$ is also compact.

Proposition 7.11. There is a $\Gamma$-equivariant homeomorphism $f: \mathrm{T}^{1} \widetilde{\Sigma} \rightarrow \mathcal{N}\left(\Lambda_{\rho}\right)$ mapping orbits of the geodesic flow $\widetilde{\Phi}^{t}$ to orbits of $\widetilde{\Phi}_{\mathcal{N}}^{t}$.

Proof. The orbit space of $\widetilde{\Phi}^{t}$ is $\partial \widetilde{\Sigma} \times \partial \widetilde{\Sigma} \backslash \mathscr{D}$, whereas the orbit space of $\widetilde{\Phi}_{\mathcal{N}}^{t}$ is $\Lambda_{\rho} \times \Lambda_{\rho} \backslash \mathscr{D}$ (where $\mathscr{D}$ denotes the diagonal in both cases). Moreover, the quotient maps $p_{\Phi}: \mathrm{T}^{1} \tilde{\Sigma} \rightarrow \partial \tilde{\Sigma} \times \partial \widetilde{\Sigma} \backslash \mathscr{D}$ and $p_{\mathcal{N}}: \mathcal{N}\left(\Lambda_{\rho}\right) \rightarrow \Lambda_{\rho} \times \Lambda_{\rho} \backslash \mathscr{D}$ are locally trivial $\mathbb{R}$-fibrations. By Proposition 7.8, there is an equivariant homeomorphism $J \times J$ between the orbit spaces; the question is to lift this homeomorphism in a $\Gamma$-equivariant way to a map $f$ so that

$$
p_{\mathcal{N}} \circ f=(j \times j) \circ p_{\Phi} .
$$

The way to perform such a lift is quite well known. Take a finite collection $\left(T_{i}\right)_{1 \leq i \leq l}$ of small transversals to $\widetilde{\Phi}^{t}$ in $\mathrm{T}^{1} \widetilde{\Sigma}$ so that for any $p$ in $\mathrm{T}^{1} \widetilde{\Sigma}$ there is a 
positive real number $t$ in ] $-1,+1\left[\right.$ such that $\Phi^{t}(p)$ lies on $\gamma T_{i}$ for some $\gamma$ in $\Gamma$. Observe that such a family is locally finite: given $x$, there are only finitely many $\gamma$ fulfilling this condition. Now, since $p_{\mathcal{N}}$ is a fibration, and if the $T_{i}$ are chosen sufficiently small, for every $i$, the restriction of $(J \times J) \circ p_{\Phi}$ to $T_{i}$ lifts to a map $\mathrm{f}_{i}: T_{i} \rightarrow \mathcal{N}\left(\Lambda_{\rho}\right)$ such that on $T_{i}$ we have

$$
p_{\mathcal{N}} \circ f_{i}=(J \times J) \circ p_{\Phi} .
$$

For every $p$ in $\mathrm{T}^{1} \widetilde{\Sigma}$, for every triple $\alpha=\left(i, \gamma, t_{i}\right)$ with $-1 \leq t_{i} \leq 1$ such that $\widetilde{\Phi}_{i}^{t}(p)$ lies in $\gamma T_{i}$ define $x_{\alpha}(p)=\rho(\gamma) \mathfrak{f}_{i}\left(\widetilde{\Phi}^{t_{i}}(p)\right)$. All these points lie on the same $\widetilde{\Phi}_{\mathcal{N}}$-orbit. Now select a partition of unity $\left.\left(f_{i}\right)_{1 \leq i \leq l}\right)$ on $N=\Gamma \backslash \mathrm{T}^{1} \widetilde{\Sigma}$ subordinate to the covering $\left.\left(U_{i}\right)_{1 \leq i \leq l}\right)$ where $U_{i}=\left\{\Phi^{t}(p) \mid-1<t<1, p \in T_{i}\right\}$. It associates to every $x_{\alpha}$ a weight, namely the value of $f_{i}$ at the projection in $N$ of $p$. Define $\mathrm{f}(p)$ as the barycenter of $x_{\alpha}$ with respect to these weights. It defines a continuous $\Gamma$-equivariant map $f$ mapping orbits of $\widetilde{\Phi}^{t}$ into orbits of $\widetilde{\Phi}_{\mathcal{N}}^{t}$. Now it follows from the hyperbolicity of $\widetilde{\Sigma}$ that a diffusion process along the orbits transform this map to another map, that we still denote $\mathfrak{f}$, which is injective along the orbits (see [Ghy84], [Gro00]). This map obviously satisfies the condition $p_{\mathcal{N}} \circ f=(J \times J) \circ p_{\Phi}$ and is $\Gamma$-equivariant. It follows that it is injective. An homological argument ensures that it is a homeomorphism.

We can now improve the content of Proposition 7.8.

Proposition 7.12. For any complete geodesic ray $\left[x_{0}, x^{+}\left[\right.\right.$in $\operatorname{Conv}\left(\Lambda_{\rho}\right)$ there is a sequence $\left(\gamma_{n}\right)_{(n \geq 1)}$ in $\Gamma$ and a convex cap $\mathfrak{H}^{+}$such that

(1) the convex caps $\mathfrak{H}_{n}^{+}:=\rho\left(\gamma_{n}\right) \mathfrak{H}^{+}$shrink uniformly to $x^{+}$,

(2) the repelling pole $x^{-}$belongs to $\mathfrak{D}^{-}:=\partial \operatorname{Conv}\left(\Lambda_{\rho}\right) \cap \mathfrak{H}^{-}$,

(3) the attracting pole $x^{+}$belongs to every $\delta_{n}^{+}:=\rho\left(\gamma_{n}\right) \mathfrak{D}^{+}$. Here by $\delta^{+}$we mean $\partial \operatorname{Conv}\left(\Lambda_{\rho}\right) \cap \mathfrak{S}^{+}$.

Proof. For every $x$ in $\left[x_{0}, x^{+}[\right.$, let $v(x)$ be the velocity, i.e., the unit vector tangent to $\left[x_{0}, x^{+}\right.$[ and oriented towards $x^{+}$. Since $\mathcal{N}(\rho)$ is compact, the $\Phi_{\mathcal{N}^{-}}^{t}$ orbit of the projection of $\left(x_{0}, v_{0}\right)$ (where $\left.v_{0}=v\left(x_{0}\right)\right)$ admits an accumulation point. Let $\left(x_{\infty}, v_{\infty}\right)$ be a lifting in $\mathcal{N}\left(\Lambda_{\rho}\right)$ of this accumulation point, and let $\mathfrak{H}^{+}$be a convex cap such that the wall $\subseteq$ intersects $\left[x_{0}, x_{+}\right.$[and such that the final extremity $\ell^{+}\left(x_{\infty}, v_{\infty}\right)$ lies in the interior of $\triangleright^{+}$. Fix also a positive real number $\varepsilon$, and let $W$ be a small neighborhood of $\left(x_{\infty}, v_{\infty}\right)$ in $\mathcal{N}\left(\Lambda_{\rho}\right)$ made of points of the form $\widetilde{\Phi}_{\mathcal{N}}^{t}(y, w)$ where $-\varepsilon<t<\varepsilon$, $y$ lies in $\mathbb{S}$, and the tangent vector $w$ points to the direction of $\mathfrak{H}^{+}$, i.e., the final extremity of the $\widetilde{\Phi}_{\mathcal{N}^{-}}^{t}$ orbit of $(y, w)$ lies in the interior of $\triangleright^{+}$.

By construction, there is a sequence $\left(\gamma_{n}\right)_{(n \in \mathbb{N})}$ and a sequence of points $x_{n}$ in $\left[x_{0}, x^{+}\right.$[ converging to the final extremity $x^{+}$such that $\left(x_{n}, v_{n}\right)$ (where $\left.v_{n}:=v\left(x_{n}\right)\right)$ intersects $\rho\left(\gamma_{n}\right) W$. By replacing $\gamma_{n}$ by $\gamma_{n} \gamma_{1}^{-1}$ and $\left(x_{\infty}, v_{\infty}\right), \mathfrak{H}^{+}$and $W$ by their images by $\rho\left(\gamma_{1}\right)$ we can assume that $\gamma_{1}$ is trivial and that $x_{1}$ belongs to $W$. Hence 
$x^{+}=\ell^{+}\left(x_{1}, v_{1}\right)$ lies in the interior of $\mathfrak{D}^{+}$. Moreover, $\rho\left(\gamma_{n}\right)^{-1} v_{n}$ points to the direction of $\mathfrak{H}^{+}$: it follows that $x^{+}$belongs to every $\mathfrak{H}_{n}^{+}$, and that $x_{0}$ belongs to $\mathfrak{H}_{n}^{-}$.

Up to a subsequence, we can assume that $\left(\rho\left(\gamma_{n}\right)\right)_{(n \in \mathbb{N})}$ is a converging subsequence with unbalanced distortion. The $\rho\left(\gamma_{n}\right) x_{\infty}$ stay at uniformly bounded distance form $x_{n}$; it follows that they converge to $x^{+}$and that $x^{+}$is the attracting pole of $\left(\rho\left(\gamma_{n}\right)\right)_{(n \in \mathbb{N})}$. On the other hand, every $\mathfrak{H}_{n}^{-}$contains $x_{0}$ : therefore, these convex caps do not shrink to a point. The repelling pole $x^{-}$lies in $\mathfrak{D}^{-}$. Hence the positive convex caps $\mathfrak{H}_{n}^{+}$shrink to the attracting pole $x^{+}$. The proposition is proved.

7.5. End of the proof of Theorem 1.2. Let $\ell_{\rho}^{ \pm}: \mathrm{T}^{1} \widetilde{\Sigma} \rightarrow \Lambda_{\rho}$ be the composition of $J: \partial \Gamma \rightarrow \Lambda_{\rho}$ with $\ell^{ \pm}: \mathrm{T}^{1} \widetilde{\Sigma} \rightarrow \partial \Gamma:$ they together define a map $\left(\ell_{\rho}^{+}, \ell_{\rho}^{-}\right): \mathrm{T}^{1} \widetilde{\Sigma} \rightarrow y$. In order to achieve the proof of the main theorem we just have to construct the metrics $g^{p}$ satisfying the hypothesis of Proposition 5.5.

Fix a $\rho(\Gamma)$-invariant future oriented time-like vector field $V$ on $E\left(\Lambda_{\rho}\right)$. For every $x$ in $\operatorname{Conv}\left(\Lambda_{\rho}\right)$ we simplify the notations by denoting simply $h^{x}$ the metric $g^{x, V(x)}$ on $\partial U(x) \subset \operatorname{Ein}_{n}$ introduced in $\S 5.2 .2$. We define $g^{x}$ as the metric $h^{\rho(\gamma) x_{0}}$ where $\gamma$ is an element of $\Gamma$ such that $\rho(\gamma) \bar{D}_{\text {conv }}(\Gamma)$ contains $x$. This family of metrics has a drawback: it is not continuous.

A way to construct a continuous family of metrics is the following. Let $\varsigma: T^{1} \widetilde{\Sigma} \rightarrow$ $\widetilde{S}_{\rho}^{+}$be the composition of the homeomorphism $\mathfrak{f}$ of Proposition 7.11 with the projection $\pi: \mathcal{N}\left(\Lambda_{\rho}\right) \rightarrow \operatorname{Conv}\left(\Lambda_{\rho}\right)$. This defines a $\Gamma$-invariant homeomorphism. For $p=(x, v)$ in $\mathrm{T}^{1} \widetilde{\Sigma}$, define a metric on the open neighborhood $\partial U(\varsigma(p))$ of $\ell_{\rho}^{+}(p)$ and $\ell_{\rho}^{-}(p)$ by $g_{0}^{p}:=h^{\varsigma}(p)$. These metrics vary continuously with $p$.

Now the key observation is that checking the expanding property for $g_{0}^{p}$ is the same as checking this property for $g^{p}$. Indeed:

Lemma 7.13. For every $\delta>0$, there is a constant $C_{\delta}>1$ such that for every $x$ and $y$ in $\operatorname{Conv}\left(\Lambda_{\rho}\right)$ such that $d^{H}(x, y)<\delta$, and for every vector $w$ tangent to $\operatorname{Ein}_{n}$ at a point of $\Lambda_{\rho}$ the following inequalities hold:

$$
C_{\delta}^{-1} h^{y}(w, w) \leq h^{x}(w, w) \leq C_{\delta} h^{y}(w, w) .
$$

Sketch of proof. When $y$ is fixed, for example, $y=x_{0}$, the lemma follows from the compactness of the $d^{H}$-ball centered at $x_{0}$ and the continuity of $x \rightarrow h^{x}$. The general case follows by $\rho(\Gamma)$-equivariance.

Hence, $g_{0}^{p}$ and $g^{p}$ only differ by a factor $C_{\delta}$ where $\delta$ is the diameter of $\bar{D}_{\text {conv }}(\Gamma)$. Therefore, the last step in the proof of Theorem 1.2 is:

Proposition 7.14. Let $p=(x, v)$ be an element of $\mathrm{T}^{1} \widetilde{\Sigma}$. Then for every $C>0$, there is a time $t>0$ such that for every tangent vector $w$ to $\operatorname{Ein}_{n}$ at $\ell_{\rho}^{+}(p)$ the inequality $g^{\widetilde{\Phi}^{t}(p)}(w, w) \geq C g^{\tilde{p}}(w, w)$ holds. 
Proof. Let $r_{0}=\left[x_{0}, x^{+}\right.$[ be the $\pi$-projection of the image by $f$ of the positive $\widetilde{\Phi}^{t}$ orbit of $p$. Observe that $x^{+}=\ell_{\rho}^{+}(p)$. Let $\mathfrak{H}^{+}$be the convex cap and $\left(\gamma_{n}\right)_{(n \geq 1)}$ be the sequence obeying the conclusion of Proposition 7.12.

According to Lemma 7.13, it is enough to prove that for every $C>0$ there is a positive integer $n$ such that the $h^{\gamma_{n} x_{0}}$-norm of any $w$ in $\mathrm{T}_{x_{+}} \operatorname{Ein}_{n}$ is bounded from below by its $h^{x_{0}}$-norm multiplied by $C$. Since the metrics are $\rho(\Gamma)$-equivariant, we have to prove that

$$
h^{x_{0}}\left(d_{x}+\rho\left(\gamma_{n}\right)^{-1} w, d_{x}+\rho\left(\gamma_{n}\right)^{-1} w\right) \geq C h^{x_{0}}(w, w) .
$$

This inequality only involves the metric $h^{x_{0}}$. But since $\Lambda_{\rho}$ is a compact subset of $\partial U\left(x_{0}\right)$, the $h^{x_{0}}$-norm of vectors tangent to points in $\Lambda_{\rho}$ is equivalent to their $\|\cdot\|_{0}$-norm; here by $\|\cdot\|_{0}$ we mean the restriction to $\operatorname{Ein}_{n}$ of the spherical metric on $\mathbb{S}\left(\mathbb{R}^{2, n}\right)$ induced by the Euclidean norm. Hence, to this end, we just have to check that Corollary 6.5 applies, i.e., that the attracting pole $x^{+}$belongs to $\rho\left(\gamma_{n}\right) D^{-}$(using the notations introduced in $\S 7.4)$.

By item (2) of Proposition 7.12, the repelling pole $x^{-}$belongs to $\mathfrak{D}^{-}$. Hence, the positive convex cap $\mathfrak{H}^{+}$is at positive distance from $\left(x^{-}\right)^{\perp}$ in the unit sphere $\mathbb{S}\left(\mathbb{R}^{2, n}\right)$, i.e., is contained in $D_{\varepsilon}$ for $\varepsilon$ sufficiently small. Hence $\rho\left(\gamma_{n}\right) D^{-}$contains $\delta_{n}^{+}$. By item (3) of Proposition 7.12, $x^{+}$lies in $\delta_{n}^{+}$. Thus we obtain that $x^{+}$belongs to $\rho\left(\gamma_{n}\right) D^{-}$, as required.

\section{Conclusion}

8.1. Closure of the set of quasi-Fuchsian representations. In the Riemannian context, the set of quasi-Fuchsian representations is not closed. But the situation for quasi-Fuchsian representations in $\mathrm{SO}_{0}(2, n)$ of lattices in $\mathrm{SO}_{0}(1, n)$ is different. While quasi-spheres in $\partial \mathbb{H}^{n+1}$ may degenerate, the limit sets of a sequence of quasi-Fuchsian representations $\left(\rho_{k}\right)_{(k \in \mathbb{N})}$ in $\mathrm{SO}_{0}(2, n)$ always converge, up to a subsequence, to a closed achronal topological sphere $\Lambda$ in $\operatorname{Ein}_{n}$. This follows from the compactness of the set of 1-Lipschitz maps $f: \mathbb{S}^{n} \rightarrow \mathbb{S}^{1}$. It is also easy to see that if the representations $\rho_{k}$ converge to a representation $\rho$, the set $\Lambda$ has to be preserved by $\rho(\Gamma)$.

\section{Question 8.1. Is $\Lambda$ acausal?}

If this question has a positive answer, the limit representation $\rho$ is quasi-Fuchsian faithfulness and discreteness follow from classical arguments. In other words, quasiFuchsian representations would form an entire component of $\operatorname{Rep}\left(\Gamma, \mathrm{SO}_{0}(2, n)\right)$.

8.2. Convex cocompact lattices. Theorem 1.1 extends, mutatis mutandis, to the case where $\Gamma$ is a non-elementary convex cocompact subgroup of $\mathrm{SO}_{0}(1, n)$, i.e., a discrete subgroup acting cocompactly on the convex hull in $\mathbb{H}^{n}$ of its limit set in 
$\partial \mathbb{H}^{n}$ (the non-elementary hypothesis meaning that we require that the cardinal of this limit set is infinite). The definition of Anosov representations can be extended in this context by considering as dynamical system $\left(N, \Phi^{t}\right)$ not the entire quotient $\Gamma \backslash \mathrm{T}^{1} \mathbb{H}^{n}$ but the non-wandering subset of the geodesic flow in this quotient. This is not anymore a manifold, but a compact lamination with a flow, namely the restriction of the geodesic flow. The set of $\left(\mathrm{SO}_{0}(2, n), y\right)$-Anosov representations is open, and it is still true that they correspond to faithful, discrete representations admitting as limit set a closed acausal subset in $\operatorname{Ein}_{n}$, but which now is not a topological sphere.

The main difference is that the associated domains $E\left(\Lambda_{\rho}\right)$ in $\operatorname{AdS}_{n+1}$ are not globally hyperbolic. However, the action of $\rho(\Gamma)$ on $E\left(\Lambda_{\rho}\right)$ is still free, properly discontinuous and strongly causal, i.e., the quotient spacetime $\rho(\Gamma) \backslash E\left(\Lambda_{\rho}\right)$ is strongly causal. In dimension $2+1$ (when $n=2$ ) these spacetimes are the so-called $B T Z$ multi-black holes (see [BTZ92, Bar08a]).

\subsection{Topology of Cauchy hypersurfaces of AdS manifolds}

8.3.1. Existence of negatively curved Cauchy hypersurfaces. In Theorem 1.2 , the group $\Gamma$ is assumed to be the fundamental group of negatively curved Riemannian manifold. Actually, it is very likely that this hypothesis can be removed, i.e., that it is automatically satisfied by GHC-regular representations. More precisely, the answer to the following question is probably positive:

Question 8.2. Let $\Gamma$ be a torsion-free group, and $\rho: \Gamma \rightarrow \operatorname{SO}_{0}(2, n)$ be a strictly GHC-regular representation. Is there a $\rho(\Gamma)$-invariant smooth (i.e., $C^{r}$ with $r \geq 2$ ) convex Cauchy hypersurface?

Here convexity means that the second fundamental form of the hypersurface is positive or negative definite, the sign depending on the orientation conventions. By the Gauss-Codazzi equations, since the ambient anti-de Sitter space has constant sectional curvature -1 , it follows that the induced metric on the hypersurface has negative curvature (more precisely, they have curvature $\leq 1$ ), and thus, $\Gamma$ is the fundamental group of a negatively curved Riemannian manifold.

Observe that we already have in hand two convex Cauchy hypersurfaces: the boundary components $S^{ \pm}$of the convex core in the associated GHC spacetime. Unfortunately these two hypersurfaces are not smooth: a possible way to give a positive answer to the question above would be to approximate $S^{ \pm}$by smooth convex hypersurfaces.

The question is more subtle that it could appear at first glance. The main task in [BBZ07] was to give a positive answer to this question in dimension $2+1$.

8.3.2. Strictly GHC-representations are weakly Anosov. Even if we still do not know the answer to Question 8.2, we can observe that $\Gamma$ is indeed negatively curved, but in a weak sense. Let $\rho: \Gamma \rightarrow \mathrm{SO}_{0}(2, n)$ be strictly GHC representation. Define 
the length of Lipschitz curves $c: I \rightarrow \widetilde{S}^{ \pm}$as the integral over $I$ of the Lorentzian norm of the tangent vector (defined almost everywhere), and then the distance $\tilde{d}^{ \pm}(x, y)$ between two points $x, y$ in $\widetilde{S}^{ \pm}$as the infimum of the length of Lipschitz curves joining $x$ to $y$. It is not hard to see that $\tilde{d}^{ \pm}$is indeed a distance, endowing $\widetilde{S}^{ \pm}$ with a length space structure.

Observe that $\left(\tilde{S}^{ \pm}, \tilde{d}^{ \pm}\right)$is not in general a Riemannian space, neither Finslerian. However, this metric structure induces the manifold topology on $\widetilde{S}^{ \pm}$, which admits a compact quotient: it is therefore a complete, proper metric space. By the generalized Hopf-Rinow Theorem ([BH99], Proposition I.3.7), $\left(\widetilde{S}^{ \pm}, \tilde{d}^{ \pm}\right)$is geodesic: for any two points $x, y$ in $\widetilde{S}^{ \pm}$, there always exists a curve realizing their distance.

Proposition 8.3. $\left(\tilde{S}^{ \pm}, \tilde{d}^{ \pm}\right)$is a complete $\mathrm{CAT}(-1)$ space.

For definition of CAT(-1) spaces, we refer to [BH99], §2.1, or [Bal95].

Proof. We only consider the upper convex boundary $\widetilde{S}^{-}$, the case of $\widetilde{S}^{+}$is similar (or obtained by reversing the time orientation). According to the Cartan-Hadamard Theorem (see e.g. [BH99], Theorem 4.1) being a CAT(-1) space is a local property. Since $\widetilde{S}^{-}$is simply connected, we just have to prove that every point $x$ admits a neighborhood where the metric $\tilde{d}^{-}$is of curvature $\leq-1$ (in the sense of [BH99], Definition II.1.2).

In the Klein model $\widetilde{S}^{-}$is locally the graph of a convex function from an open domain of $\mathbb{R}^{n}$ into $\mathbb{R}$. More precisely, there is a coordinate system $\left(t, \bar{x}_{1}, \ldots, \bar{x}_{n}\right)$, $-\varepsilon<x_{i}<\varepsilon,-\eta<t<\eta$, on a neighborhood $U$ of $x$ such that

- $x$ has coordinates $(0, \ldots, 0)$,

- $U \cap \widetilde{S}^{-}$is the graph of a convex map $\left.\psi:\right]-\varepsilon, \varepsilon\left[^{n} \rightarrow\right]-\eta, \eta[$,

- $\{t=0\}$ is a support hyperplane for $\psi$,

- every tangent vector with negative norm for $-d \eta^{2}+d x_{1}^{2}+\cdots+d x_{n}^{2}$ has negative norm for the AdS metric.

Shrinking $\varepsilon$ if necessary, we moreover can assume that the gradient of $\psi$ has almost everywhere $d x_{1}^{2}+\cdots+d x_{n}^{2}$-norm less than 1 . By convolution, we obtain smooth convex maps $\psi_{v}$ which uniformly converge to $\psi$ when the parameter $v>0$ converges to 0 . Moreover, the norm of their gradient is bounded from above by 1 , it follows that the graphs $S_{v}$ of $\psi_{v}$ are space-like. Finally, this uniform convergence implies that for any Lipschitz curve $c: I \rightarrow]-\varepsilon, \varepsilon\left[{ }^{n}\right.$, the AdS length of $s \rightarrow\left(c(s), \psi_{\nu}(c(s))\right.$ uniformly converges to the AdS-length of $s \rightarrow\left(c(s), \psi(c(s))\right.$. Hence the graphs $S_{v}$, equipped with their induced (Riemannian) length metric, converge in the GromovHausdorff topology to the restriction of $\tilde{d}^{-}$to $U \cap \widetilde{S}^{-}$(cf. [BH99, Definition I.5.33]).

Since each $S_{v}$ is smooth and convex, as we already noticed, it has curvature $\leq-1$, and is thus CAT $(-1)$. The proposition follows since Gromov-Hausdorff limits of CAT(-1) length spaces are CAT(-1) spaces ([BH99], Theorem II.3.9). 
CAT $(-1)$ spaces enjoy many nice properties. For example, they are hyperbolic in the sense of Gromov; hence the group $\Gamma$ is Gromov hyperbolic. Furthermore:

Corollary 8.4 (Proposition II.2.2 in [BH99]). ( $\left.\widetilde{S}^{ \pm}, \tilde{d}^{ \pm}\right)$is uniquely geodesic: given two points $x, y$ there is an unique geodesic joining them.

We therefore can define the geodesic flow of $S^{ \pm}$, even if $S^{ \pm}$has no unit tangent bundle.

Definition 8.5. Let $\widetilde{G}^{ \pm}$denote the space of complete unit speed geodesics of $\widetilde{S}^{ \pm}$, i.e., isometries $c: \mathbb{R} \rightarrow \widetilde{S}^{ \pm}$, endowed with the topology of uniform convergence on compact subsets. The geodesic flow $\widetilde{\Phi}_{ \pm}^{t}$ is the flow defined by

$$
\widetilde{\Phi}_{ \pm}^{t}(c)(s)=c(s+t)
$$

The group $\rho(\Gamma)$ acts naturally, freely and properly discontinuously on $\widetilde{\sigma}^{ \pm}$. We denote by $\varsigma_{\rho}^{ \pm}$the quotient space, and by $\Phi_{ \pm}^{t}$ the flow on $\varsigma_{\rho}^{ \pm}$induced by $\widetilde{\Phi}_{ \pm}^{t}$.

This flow is not differentiable but weakly (or topologically) Anosov: there are two continuous $\Gamma$-invariant foliations $\widetilde{\mathcal{F}}_{ \pm}^{s}, \widetilde{\mathcal{F}}_{ \pm}^{u}$ on $\widetilde{\widetilde{F}}^{ \pm}$, invariant by the geodesic flow such that for every pair $p, q$ of points in the same leaf of $\widetilde{\mathcal{F}}^{s}$ (respectively $\tilde{\mathcal{F}}^{u}$ ) there is a real number $t_{0}$ such that the distance between $\widetilde{\Phi}_{ \pm}^{t+t_{0}}(p)$ and $\widetilde{\Phi}_{ \pm}^{t}(q)$ decreases (respectively increases) exponentially with $t$. This claim follows quite easily from the CAT $(-1)$ property; it is actually a general property of Gromov hyperbolic spaces admitting compact quotients: see [Gro], § 8.3, and for more details, [Cha94], [Mat]. The fact that the spaces we consider are CAT $(-1)$ greatly simplifies the definition of the geodesic flow.

It should be clear to the reader that the methods used in the present paper prove that the strictly GHC-regular representation $\rho$ satisfies the $\left(\mathrm{SO}_{0}(2, n), y\right)$-Anosov property as defined in Remark 5.4 or appearing as hypothesis in Proposition 5.5; observe that in these formulations the differential of the flow is not involved. The arguments in $\S 5.3$ still apply for this non-differentiable version of $\left(\mathrm{SO}_{0}(2, n), \mathcal{Y}\right)$-Anosov property. In other words, we can state, with no more a priori restriction on the group $\Gamma$, that GHC-regular representations with acausal limit sets are precisely weakly $\left(\mathrm{SO}_{0}(2, n), y\right)$-Anosov representations. Moreover, we guess that weakly Anosov representations form an open subset of $\operatorname{Rep}(\Gamma, G)$ : the differentiable setting could probably be avoided through arguments in [Sul85]. For the pair $\left(\mathrm{SO}_{0}(2, n), y\right)$, it follows from the discussion above and from the fact that GHC-regular representations form an open domain. This latter fact follows from arguments given in [Mes07], which show that small deformations of holonomy representations of MGHC AdSspacetimes are still holonomy representations of MGHC spacetimes. The introduction of [Bon05] gives more details on this question.

Finally, as before, we can ask the question: 
Question 8.6. Is the space of (weakly) $\left(\mathrm{SO}_{0}(2, n), y\right)$-Anosov representations closed?

As in the case where $\Gamma$ is a lattice of $\mathrm{SO}_{0}(1, n)$, this essentially reduces to the proof that the limit set of a sequence of $\left(\mathrm{SO}_{0}(2, n), y\right)$-Anosov representations is acausal.

8.3.3. Classification of MGHC spacetimes of constant curvature -1. Actually, we can even wonder if the following strong version of Question 8.2 is true:

Question 8.7. Let $\rho: \Gamma \rightarrow \mathrm{SO}_{0}(2, n)$ be a strictly GHC-representation. Is it a quasi-Fuchsian representation, i.e., is $\Gamma$ isomorphic to a lattice of $\mathrm{SO}_{0}(1, n)$ ?

If the answer to this question were positive, Theorem 1.1 would not merely be a particular case of Theorem 1.2 but would correspond to the general case.

A natural way to find a positive answer to this question is to exhibit in the associated MGHC spacetime a Cauchy hypersurface with constant Gauss curvature -1 : one of the main results of [BBZ] is precisely that such a Cauchy hypersurface exists in the $(2+1)$-dimensional case. Of course, in this dimension it is only a matter to prove that the genus of the Cauchy surfaces is $\geq 2$, which can be obtained with more elementary arguments. However, this approach does not extends in higher dimension (unlike most part of the content of [BBZ]).

Another way to give a positive answer would be to study the functional on $\operatorname{Anos} y\left(\Gamma, \mathrm{SO}_{0}(2, n)\right)$ associating to a representation the volume of the convex core in the associated spacetime. Indeed, according to Lemma 3.13, this functional vanishes only on Fuchsian representations.

Finally, it is easy to produce GHC-regular representations with non-acausal limit set: let $(p, q)$ be a pair of positive integers such that $p+q=n$, and let $\Gamma$ be a cocompact lattice of $\mathrm{SO}_{0}(1, p) \times \mathrm{SO}_{0}(1, q)$. There is a natural inclusion of $\mathrm{SO}_{0}(1, p) \times \mathrm{SO}_{0}(1, q)$ into $\mathrm{SO}_{0}(2, n)$ arising from the orthogonal splitting $\mathbb{R}^{2, n}=$ $\mathbb{R}^{1, p} \oplus \mathbb{R}^{1, p}$. The isotropic cone of $\mathbb{R}^{1, p}$ (respectively $\mathbb{R}^{1, q}$ ) is contained in $\bigodot_{n}$ and its projection in $\operatorname{Ein}_{n}$ is the union of two space-like spheres $\Lambda_{p}^{ \pm} \approx \mathbb{S}^{p-1}$ (respectively $\Lambda_{q}^{ \pm} \approx \mathbb{S}^{q-1}$ ). Every point in $\Lambda_{p}^{ \pm}$is joined to every point in $\Lambda_{q}^{ \pm}$by a light-like geodesic segment in $\operatorname{Ein}_{n}$. Let $\Lambda$ be the union of light-like geodesic segments joining a point of $\Lambda_{p}^{+}$to a point in $\Lambda_{q}^{+}$and avoiding $\Lambda_{p}^{-} \cup \Lambda_{q}^{-}$. The proofs of the following facts are left to the reader:

$\Lambda$ is a non-purely light-like achronal topological sphere.

The convex hull Conv $(\Lambda)$ of $\Lambda$ in $\operatorname{AdS}_{n+1}$ coincide with the regular domain $E(\Lambda)$.

The group $\Gamma \subset \mathrm{SO}_{0}(1, p) \times \mathrm{SO}_{0}(1, q) \subset \mathrm{SO}_{0}(2, n)$ preserves the convex hull $\operatorname{Conv}(\Lambda)=E(\Lambda)$, and the quotient spacetime $M(\Gamma)=\Gamma \backslash E(\Lambda)$ is MGH. Moreover, it is spatially compact: indeed, the set of orthogonal sums $u+v$ where $u$ (respectively $v$ ) is an element of $\mathbb{R}^{1, p}$ such that $\mathrm{q}_{1, p}(u)=-1 / 2$ (respectively an element of $\mathbb{R}^{1, q}$ 
of $\mathrm{q}_{1, q}$-norm -1/2) admits two components in $\operatorname{AdS}_{n+1}$, one of which lies in $E(\Lambda)$. This component is a space-like hypersurface isometric to $\mathbb{H}^{p} \times \mathbb{H}^{q}$ and is $\Gamma$-invariant. Its projection in the quotient $M(\Lambda)$ is a compact space-like hypersurface, hence a Cauchy hypersurface.

Remark 8.8. By the Margulis Superrigidity Theorem ([Mar91]), if $p, q \geq 2$, then every $\Gamma$ into $\mathrm{SO}_{0}(2, n)$ either has finite image, or is conjugate in $\mathrm{SO}_{0}(2, n)$ to the inclusion $\Gamma \subset \mathrm{SO}_{0}(1, p) \times \mathrm{SO}_{0}(1, q) \subset \mathrm{SO}_{0}(2, n)$. It follows that every MGHC spacetime of constant curvature -1 with fundamental group isomorphic to a lattice $\Gamma$ in $\mathrm{SO}_{0}(1, p) \times \mathrm{SO}_{0}(1, q)$ is isometric to a spacetime $M(\Gamma)$ described above.

Remark 8.9. When $n=2$, the only possibility is $p=q=1$. This is the case of torus universe (see [BBZ07], § 7, and [Car03], § 3.3).

Question 8.10. Let $\rho: \Gamma \rightarrow \mathrm{SO}_{0}(2, n)$ be a GHC-regular representation with nonacausal limit set. Is $\Gamma$ isomorphic to a lattice of some product $\mathrm{SO}_{0}(1, p) \times \mathrm{SO}_{0}(1, q)$ ?

Our personal guess is that all the questions listed above admit a positive answer.

Conjecture 8.11. Every GHC-regular representation into $\mathrm{SO}_{0}(2, n)$ is either a quasiFuchsian representation of a lattice in $\mathrm{SO}_{0}(1, n)$, or a representation of a lattice in $\mathrm{SO}_{0}(1, p) \times \mathrm{SO}_{0}(1, q)$ with $p+q=n, p \geq 1, q \geq 1$.

\section{References}

$\left[\mathrm{ABB}^{+}\right.$07] L. Andersson, T. Barbot, R. Benedetti, F. Bonsante, W. M. Goldman, F. Labourie, K. P. Scannell, and J.-M. Schlenker, Notes on a paper of Mess. Geom. Dedicata 126 (2007), 47-70. Zbl 1126.53042 MR 2328922

[AGH98] L. Andersson, G. J. Galloway, and R. Howard, The cosmological time function. Classical Quantum Gravity 15 (1998), 309-322. Zbl 0911.53039 MR 1606594

[Bal95] W. Ballmann, Lectures on spaces of nonpositive curvature. DMV Seminar 25, Birkhäuser, Basel 1995. Zbl 0834.53003 MR 1377265

[BTZ92] M. Bañados, C. Teitelboim, and J. Zanelli, Black hole in three-dimensional spacetime. Phys. Rev. Lett. 69 (1992), 1849-1851. Zbl 0968.83514 MR 1181663

[Bar08b] T. Barbot, Causal properties of AdS-isometry groups. I. Causal actions and limit sets. Adv. Theor. Math. Phys. 12 (2008), 1-66. Zbl 1151.83311 MR 2369412

[Bar08a] T. Barbot, Causal properties of AdS-isometry groups. II. BTZ multi-black-holes. Adv. Theor. Math. Phys. 12 (2008), 1209-1257. Zbl 1153.83349 MR 2443264

[Bar10] T. Barbot, Three-dimensional Anosov flag manifolds. Geom. Topol. 14 (2010), 153-191. Zbl 1177.57011 MR 2578303

[BBZ07] T. Barbot, F. Béguin, and A. Zeghib, Constant mean curvature foliations of globally hyperbolic spacetimes locally modelled on $\mathrm{AdS}_{3}$. Geom. Dedicata 126 (2007), 71-129. Zbl 05200417 MR 2328923 
[BBZ] T. Barbot, F. Béguin, and A. Zeghib, Prescribing Gauss curvature of surfaces in 3dimensional spacetimes: application to the Minkowski problem in the Minkowski space. Ann. Inst. Fourier (Grenoble) 61 (2011), 511-591. Zbl 1234.53019 MR 2895066

$\left[\mathrm{BCD}^{+} 08\right]$ T. Barbot, V. Charette, T. Drumm, W. M. Goldman, and K. Melnick, A primer on the $(2+1)$ Einstein universe. In Recent developments in pseudo-Riemannian geometry, ESI Lect. Math. Phys., Eur. Math. Soc., Zürich 2008, 179-229. Zbl 1154.53047 MR 2436232

[BEE96] J. K. Beem, P. E. Ehrlich, and K. L. Easley, Global Lorentzian geometry. Monographs Textbooks Pure Appl. Math. 202, 2nd ed., Marcel Dekker, New York 1996. Zbl 0846.53001 MR 1384756

[BB09] R. Benedetti and F. Bonsante, Canonical Wick rotations in 3-dimensional gravity. Mem. Amer. Math. Soc. 198 (2009), no. 926. Zbl 1165.53047 MR 2499272

[BH99] M. R. Bridson and A. Haefliger, Metric spaces of non-positive curvature. Grundlehren Math. Wiss. 319, Springer-Verlag, Berlin 1999. Zbl 0988.53001 MR 1744486

[BLIW05] M. Burger, A. Iozzi, F. Labourie, and A. Wienhard, Maximal representations of surface groups: symplectic Anosov structures. Pure Appl. Math. Q. 1 (2005), 543-590. Zbl 1157.53025 MR 2201327

[BK53] H. Busemann and P. J. Kelly, Projective geometry and projective metrics. Pure Appl. Math. 3, Academic Press, New York 1953. Zbl 0052.37305 MR 0054980

[Bon05] F. Bonsante, Deforming the Minkowskian cone of a closed hyperbolic manifold. Ph.D. thesis, Pisa 2005.

[Car03] S. Carlip, Quantum gravity in $2+1$ dimensions. Cambridge Monographs Math. Phys., Cambridge University Press, Cambridge 1998. Zbl 0919.53024 MR 1637718

[Cha94] C. Champetier, Petite simplification dans les groupes hyperboliques. Ann. Fac. Sci. Toulouse Math. (6) 3 (1994), 161-221. Zbl 0803.53026 MR 1283206

[Fra05] C. Frances, Lorentzian Kleinian groups. Comment. Math. Helv. 80 (2005), 883-910. Zbl 1083.22007 MR 2182704

[Ghy84] É. Ghys, Flots d'Anosov sur les 3-variétés fibrées en cercles. Ergodic Theory Dynam. Systems 4 (1984), 67-80. Zbl 0527.58030 MR 758894

$\left[\mathrm{GdLH}^{+} 90\right]$ E. Ghys, P. de la Harpe (Eds.), Sur les groupes hyperboliques d'après Mikhael Gromov. Progr. Math. 83, Birkhäuser, Boston 1990. Zbl 0731.20025 MR

[Gro00] M. Gromov, Three remarks on geodesic dynamics and fundamental group. Enseign. Math. (2) 46 (2000), 391-402. Zbl 1002.53028 MR 1805410

[Gro] Hyperbolic groups. In Essays in group theory, Math. Sci. Res. Inst. Publ. 8, Springer-Verlag, New York 1987, 75-263. Zbl 0634.20015 MR 0919829

[Gui08] O. Guichard, Composantes de Hitchin et représentations hyperconvexes de groupes de surface. J. Differential Geom. 80 (2008), 391-431. Zbl 1223.57015 MR 2472478

[Lab06] F. Labourie, Anosov flows, surface groups and curves in projective space. Invent. Math. 165 (2006), 51-114. Zbl 1103.32007 MR 2221137 
[Mar91] G. A. Margulis, Discrete subgroups of semisimple Lie groups. Ergeb. Math. Grenzgeb. (3) 17, Springer-Verlag, Berlin 1991. Zbl 0732.22008 MR 1090825

[Mat] F. Matthéus, Flot géodésique et groupes hyperboliques d'après M. Gromov. Sémin. Théor. Spectr. Géom. 9 (1990-1991), 67--87. http://www.numdam.org/item?id=TSG_1990-1991_9_67_0

[Mes07] G. Mess, Lorentz spacetimes of constant curvature. Geom. Dedicata 126 (2007), 3-45. Zbl 1206.83117 MR 2328921

[O’N83] B. O’Neill, Semi-Riemannian geometry. Pure Appl. Math. 103, Academic Press, New York 1983. Zbl 0531.53051 MR 719023

[Rat06] J. G. Ratcliffe, Foundations of hyperbolic manifolds. Graduate Texts in Math. 149, 2nd ed., Springer, New York 2006. Zbl 1106.51009 MR 2249478

[Sal99] F. Salein, Variétés anti-de Sitter de dimension 3. Ph.D. thesis, École Normale Sup. de Lyon, Lyon 1999.

[Sul85] D. Sullivan, Quasiconformal homeomorphisms and dynamics II: Structural stability implies hyperbolicity for Kleinian groups. Acta Math. 155 (1985), 243-260. Zbl 0606.30044 MR 806415

Received August 13, 2009; revised November 13, 2010

T. Barbot, École Normale Supérieure de Lyon, CNRS UMR 5669, 46, allée d'Italie, 69364 Lyon Cedex 07, France

E-mail: Thierry.Barbot@univ-avignon.fr

Q. Mérigot, Laboratoire Jean Kuntzmann, Université Joseph Fourier, Campus de Saint Martin d'Hères BP 53, 38041 Grenoble Cedex 09, France

E-mail: Quentin.Merigot@imag.fr 